\title{
Plurisubharmonic functions and their singularities
}

\author{
Christer O. KISELMAN \\ Uppsala University, Department of Mathematics \\ P. O. Box 480, S-751 06 Uppsala, Sweden \\ E-mail: cok@math.uu.se \\ Resumo \\ Plursubharmonaj funkcioj kaj iliaj malregulejoj
}

La temo de tiuj ci lekcioj estas lokaj kaj mallokaj ecoj de plursubharmonaj funkcioj. Unue diferencialaj neegalajoj difinantaj konveksajn, subharmonajn kaj plursubharmonajn funkciojn estas pritraktitaj. Estas pruvite ke la marĝena funkcio de plursubharmona funkcio estas plursubharmona sub certaj supozoj. Ni studas la malregulejojn de plursubharmonaj funkcioj per metodoj de la teorio pri konvekseco. En la lasta capitro ni pliĝeneraligas la klasikajn nociojn de ordo kaj tipo de entjera funkcio de finia ordo al kiom ajn rapide kreskantaj funkcioj.

\begin{abstract}
The theme of these lectures is local and global properties of plurisubharmonic functions. First differential inequalities defining convex, subharmonic and plurisubharmonic functions are discussed. It is proved that the marginal function of a plurisubharmonic function is plurisubharmonic under certain hypotheses. We study the singularities of plurisubharmonic functions using methods from convexity theory. Then in the final chapter we generalize the classical notions of order and type of an entire function of finite order to functions of arbitrarily fast growth.
\end{abstract}

This work was partially supported by the Swedish Natural Science Research Council. 


\section{Contents} \\ Introduction \\ Chapter 1. Convexity and plurisubharmonicity \\ 1.1. Introduction \\ 1.2. Conditions on the derivatives of convex and plurisubharmonic functions \\ 1.3. The minimum principle \\ Chapter 2. The Lelong number and the integrability index \\ 2.1. Introduction \\ 2.2. Spherical means and spherical suprema \\ 2.3. The Hörmander-Bombieri theorem and the integrability index \\ 2.4. Analyticity theorems for sets of plurisubharmonic functions \\ Chapter 3. Order and type as measures of growth \\ 3.1. Introduction \\ 3.2. Order and type in classical complex analysis \\ 3.3. Relative order and type of convex functions \\ 3.4. Order and type in duality \\ 3.5. The infimal convolution \\ 3.6. The order of an entire function \\ 3.7. A geometric characterization of the relative order \\ 3.8. An extension theorem for holomorphic functions \\ References
}

\title{
Introduction
}

The plurisubharmonic functions appear in complex analysis as logarithms of moduli of holomorphic functions and as analogues of potentials. Their usefulness for many constructions is due to the fact that they are easier to manipulate than holomorphic functions - this is why Lelong [1985] includes them among "les objets souples de l'analyse complexe."

In these lectures we shall first consider analogies between the convex, subharmonic, and plurisubharmonic functions: these three classes can be defined using differential inequalities. We shall also study marginal functions of plurisubharmonic functions, i.e., functions of the form

$$
g\left(x_{1}, \ldots, x_{n}\right)=\inf _{y_{1}, \ldots, y_{m}} f\left(x_{1}, \ldots, x_{n}, y_{1}, \ldots, y_{m}\right) .
$$

It is a known fact that marginal functions of convex functions are convex, but the corresponding result is not true for plurisubharmonic functions. However, it is true under some extra hypotheses, and we shall establish one such result, called the minimum principle, in Chapter 1.

In Chapter 2, we use the minimum principle to prove that sets related to plurisubharmonic functions are analytic varieties. The model result here is Siu's theorem, which says that the set of points where the Lelong number is larger than or equal to a certain number is an analytic variety. We shall see that the minimum principle provides us with a family of plurisubharmonic functions related to a given one, and that there are analyticity theorems for families of plurisubharmonic functions which are easy to deduce from the HörmanderBombieri theorem.

In the third chapter we shall take a look at the classical notions of order and type for entire functions. To every entire function $F$ we can in a natural way associate a convex 
function $f$ which describes its growth:

$$
f(t)=\sup _{|z|=e^{t}} \log |F(z)|, \quad t \in \mathbf{R} .
$$

We call $f$ the growth function of $F$. That $f$ is convex is the content of Hadamard's threecircle theorem. These classical definitions can quite naturally be extended to plurisubharmonic functions; just replace $\log |F|$ by an arbitrary plurisubharmonic function. What we do in classical complex analysis is to compare the growth of two convex functions, the growth function $f$ and the growth function $g(t)=e^{t}$ of the exponential function $G(z)=e^{z}$. The notion of relative order, the order of $f$ relative to $g$, arises from such a comparison of two convex functions. The notion of relative type of one function with respect to another is the result of a slightly different comparison.

All classical results on order and type can now be considered in this more general setting, and many of them have very precise counterparts. It should be stressed that the functions we consider may grow arbitrarily fast, whereas classically one considers functions of finite order. We have adjusted the definitions so that order and type become dual in the sense of convexity theory. This fact is very useful, for we can often choose to do calculations either on the functions themselves or on their conjugate functions, their Fenchel transforms.

The relative order determines the maximal domain in which a solution to a natural extension problem exists. This extension problem can be formulated for convex, plurisubharmonic or entire functions - the resulting domain of existence is the same in all three cases.

Acknowledgments. I am grateful to the Séminaire de Mathématiques Supérieures for the invitation to participate in this summer school. It was a great experience! It is also a pleasure to acknowledge the good help provided by Stefan Halvarsson, who have typed Chapter 1 into $\mathrm{T}_{\mathrm{E}} \mathrm{X}$, made many useful suggestions, and proofread all the chapters. My thanks go also to Maciej Klimek for checking the manuscript and for valuable comments on the presentation. Chapter 3 is essentially taken from my paper [1993] (which contains four additional sections). The London Mathematical Society has kindly given its permission to include this material here. 


\section{Chapter 1. Convexity and plurisubharmonicity}

\subsection{Introduction}

Let us first recall that the real-valued convex functions on the real line are those that satisfy the inequality

$$
f((1-t) x+t y) \leqslant(1-t) f(x)+t f(y), \quad 0 \leqslant t \leqslant 1, x, y \in \mathbf{R} .
$$

In particular, for $t=1 / 2$ they satisfy

$$
f(c) \leqslant \frac{1}{2} f(c-r)+\frac{1}{2} f(c+r), \quad c, r \in \mathbf{R},
$$

which can be written as

$$
f(c) \leqslant M_{\partial I} f
$$

denoting by $M$ the mean value over a set, in this case $\partial I=\{c-r, c+r\}$, which is the boundary of the one-dimensional ball $c+r B$.

Some regularity has to be imposed if we use (1.1.2) though, for while (1.1.1) implies that $f$ is continuous (where it is real-valued), (1.1.2) does not:

Example. Take a Hamel basis for the vector space of all real numbers over the rational numbers with 1 and $\sqrt{2}$ as basis elements. Define $f$ to be a $\mathbf{Q}$-linear form $f: \mathbf{R} \rightarrow \mathbf{Q}$ such that $f(1)=1, f(\sqrt{2})=0$. Then obviously $f$ satisfies (1.1.1) for rational $t$ (with equality), in particular (1.1.2), but it is not continuous (and we would not like to call it convex). Indeed, $f(s+t \sqrt{2})=s$ for rational $s, t$, which shows that $f$ is unbounded near any point.

However, (1.1.2) plus some mild regularity assumption (like semicontinuity or even measurability) is equivalent to (1.1.1) for real-valued functions.

The definition of a subharmonic function is a generalization of this: a function $f$ is called subharmonic in an open subset $\Omega$ of $\mathbf{R}^{n}$ if it takes its values in $[-\infty,+\infty$, is upper semicontinuous, and satisfies the mean-value inequality

$$
f(c) \leqslant M_{\partial A} f
$$

whenever $A$ is a closed ball of center $c$ contained in $\Omega \subset \mathbf{R}^{n}$. We shall write $f \in S H(\Omega)$. The constant $-\infty$ is allowed.

However, we can generalize the notion of a convex function of one variable in a different direction: we consider a function in $\mathbf{R}^{n}$ and look at its restrictions to real lines, in other words at its pull-backs $\varphi^{*} f=f \circ \varphi$ for an arbitrary affine function $\varphi: \mathbf{R} \rightarrow \mathbf{R}^{n}$. If this pull-back is always convex, then $f$ is called convex in $\mathbf{R}^{n}$. (Actually such a function should be called "pluriconvex" if we were to follow the idea that has led to the word plurisubharmonic!) We shall write $f \in C V X(\Omega)$ if $f$ is real-valued and convex in a convex open set $\Omega$. 
Remark. In convexity theory one usually allows values in $[-\infty,+\infty]$. A function $f: \mathbf{R}^{n} \rightarrow$ $[-\infty,+\infty]$ is defined to be convex if its epigraph

$$
\text { epi } f=\left\{(x, t) \in \mathbf{R}^{n} \times \mathbf{R} ; f(x) \leqslant t\right\}
$$

is convex as a subset of $\mathbf{R}^{n} \times \mathbf{R}$. It is sometimes more convenient to use the strict epigraph

$$
\operatorname{epi}_{s} f=\left\{(x, t) \in \mathbf{R}^{n} \times \mathbf{R} ; f(x)<t\right\} .
$$

It is easy to see that the epigraph and the strict epigraph are convex simultaneously. For real-valued functions, the definition using the epigraph is equivalent to (1.1.1).

We can now generalize the subharmonic functions of one complex variable in the same way as we did when we defined convex functions in $\mathbf{R}^{n}$. If $\varphi^{*} f=f \circ \varphi$ is subharmonic for all complex affine mappings $\varphi: \mathbf{C} \rightarrow \mathbf{C}^{n}$ and has in addition some kind of regularity, then $f$ is called plurisubharmonic. The additional regularity assumption is usually taken to be upper semicontinuity, which means the the strict epigraph epi $f$ (cf. (1.1.4)) is assumed to be open.

Definition 1.1.1. We say that $f$ is plurisubharmonic in an open set $\Omega$ in $\mathbf{C}^{n}$ if $f: \Omega \rightarrow\left[-\infty,+\infty\left[\right.\right.$ is upper semicontinuous in $\Omega$ and, for all $a, b \in \mathbf{C}^{n}, z \mapsto f(a+z b)$ is subharmonic as a function of the complex variable $z$ in the open set where it is defined. Notation: $f \in P S H(\Omega)$.

The scheme of generalizations can be illustrated as follows:

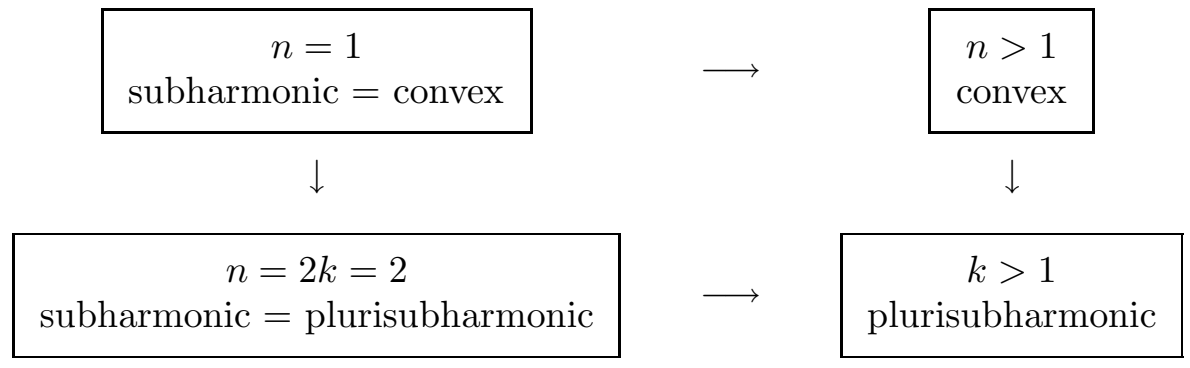

In all cases, the mean-value inequality $f(c) \leqslant M_{\partial A} f$ is imposed, but with different balls $A$ : they can be real one-dimensional or complex one-dimensional or full-dimensional. This will lead to important analogies between the different cones of functions: the cone $P S H$ is sometimes analogous with $S H$, sometimes with $C V X$.

A very natural question is this: if the pull-back $\varphi^{*} f$ is subharmonic for all affine functions $\varphi$ mapping the complex plane into $\mathbf{C}^{n}$, is $f$ plurisubharmonic? In other words, is the assumption of upper semicontinuity superfluous? The answer seems to be unknown. There is a similar question whether separately subharmonic ${ }^{1}$ functions are subharmonic: this is not true as shown by Wiegerinck [1988]. However, if we add some, even very weak, integrability condition, separately subharmonic functions are indeed subharmonic; see Riihentaus [1989].

It is not difficult to prove the following inclusions:

$$
C V X_{l o c}(\Omega) \subset S H(\Omega), \quad \Omega \subset \mathbf{R}^{n},
$$

${ }^{1}$ This means that the function is subharmonic in each variable when the others are kept fixed. 
and

$$
C V X_{l o c}(\Omega) \subset P S H(\Omega) \subset S H(\Omega), \quad \Omega \subset \mathbf{C}^{n},
$$

where $C V X_{l o c}(\Omega)$ is the cone of functions which are locally convex, i.e., convex in some ball around an arbitrary point. They can be proved using the mean-value inequalities, but they will also follow from the differential inequalities to be presented in the next section.

For general information about plurisubharmonic functions see Hörmander [1990; forthc.], Klimek [1991], and Lelong [1969].

\subsection{Conditions on the derivatives of convex and plurisubharmonic functions}

We shall now take a look at various differential inequalities which are related to convexity, subharmonicity and plurisubharmonicity. The simplest is this:

Proposition 1.2.1. Let $f \in C^{2}(I)$, where $I \subset \mathbf{R}$ is an interval. Then $f$ is convex if and only if $f^{\prime \prime} \geqslant 0$.

This can of course be proved directly, but since it is a special case of Proposition 1.2.3 below, we omit the proof.

We shall write $\mathcal{D}(\Omega)$ for the set of all test functions in an open set $\Omega$ and $\mathcal{D}^{\prime}(\Omega)$ for the set of all distributions in $\Omega$, the space dual to $\mathcal{D}(\Omega)$.

Proposition 1.2.2. Let $f \in L_{l o c}^{1}(I), I$ being an interval. Then $f$ is equal to a convex function almost everywhere if and only if $f^{\prime \prime} \geqslant 0$ in the sense of distributions, i.e., $\int_{I} \varphi^{\prime \prime} f d \lambda \geqslant 0$ for all $\varphi \in \mathcal{D}(I)$ satisfying $\varphi \geqslant 0$. Moreover, if $u$ is a distribution in $I, u \in \mathcal{D}^{\prime}(I)$, then there exists a convex function $f$ such that $\int_{I} f \varphi d \lambda=u(\varphi)$ for every test function $\varphi \in \mathcal{D}(I)$ if and only if $u^{\prime \prime} \geqslant 0$.

This result is a special case of Proposition 1.2.4 below.

Proposition 1.2.3. Let $f \in C^{2}(\Omega), \Omega \subset \mathbf{R}^{n}$. Then $f \in S H(\Omega)$ if and only if $\Delta f \geqslant 0$, where $\Delta=\partial^{2} / \partial x_{1}^{2}+\cdots+\partial^{2} / \partial x_{n}^{2}$ is the Laplacian.

Proof. We shall write $B$ for the closed unit ball and $S$ for its boundary, the unit sphere, so that $c+r B$ is the closed ball of radius $r$ and center at $c$, and $c+r S$ its boundary. Let $E$ be the fundamental solution of the Laplacian such that $\Delta E=\delta_{c}$ and $E$ vanishes on the sphere $c+r S$. Then Green's formula yields

$$
f(c)-f_{c+r S} f=\int_{c+r B} E \Delta f d \lambda,
$$

where $d \lambda$ denotes Lebesgue measure. We use a barred integral sign to denote mean value, thus

$$
M_{A}(f)=f_{A} f d \lambda=\int_{A} f d \lambda / \int_{A} d \lambda \text { provided } 0<\int_{A} d \lambda<+\infty .
$$

Since $E \leqslant 0$ in the ball $x+r B, \Delta f \geqslant 0$ implies

$$
f(c)-f_{c+r S} f \leqslant 0 .
$$


This holds for all $c \in \Omega$ and all $r$ such that $c+r B \subset \Omega$. This is the mean-value inequality for $f$.

For the other direction, assume $\Delta f(c)<0$ at some point $c$. Take $r$ so small that $\Delta f<0$ in $c+r B$. Then (1.2.1) shows that

$$
f(c)-f_{c+r S} f>0
$$

for these $r$, so $f$ does not satisfy the mean-value inequality.

Proposition 1.2.4. Let $u \in \mathcal{D}^{\prime}(\Omega), \Omega \subset \mathbf{R}^{n}$. Then there exists $f \in S H(\Omega)$ such that $\int f \varphi d \lambda=u(\varphi)$ for all $\varphi \in \mathcal{D}(\Omega)$ if and only if $\Delta u \geqslant 0$ in the sense of distributions, i.e., $u(\Delta \varphi) \geqslant 0$ for all $\varphi \in \mathcal{D}(\Omega)$ satisfying $\varphi \geqslant 0$.

Proof. First let $f \in S H(\Omega)$. Form $f_{\varepsilon} \in C^{\infty}\left(\Omega_{\varepsilon}\right)$ by convolution:

$$
f_{\varepsilon}(x)=\left(f * \psi_{\varepsilon}\right)(x)=\int f(y) \psi_{\varepsilon}(x-y) d \lambda(y)=\int f(x-\varepsilon y) \psi(y) d \lambda(y), \quad x \in \mathbf{R}^{n},
$$

where $\psi$ is a radial ${ }^{2} C^{\infty}$ function with support in the unit ball and of integral one satisfying $\psi \geqslant 0$, and $\psi_{\varepsilon}(x)=\varepsilon^{-n} \psi(x / \varepsilon)$. Then $f_{\varepsilon}$ is subharmonic in $\Omega_{\varepsilon}=\{x \in \Omega ; x+\varepsilon B \subset \Omega\}$. Indeed, the integral $\int f(x-\varepsilon y) \psi(y) d \lambda(y)$ is a limit of finite sums $\sum f\left(x-\varepsilon y^{j}\right) c_{j}$ with positive $c_{j}$. Since $f_{\varepsilon}$ is smooth, Proposition 1.2.3 implies that $\Delta f_{\varepsilon} \geqslant 0$. When $\varepsilon \rightarrow 0, f_{\varepsilon}$ tends to $f$ in $L_{l o c}^{1}(\Omega)$ and the positivity in the sense of distributions is preserved: $\Delta f \geqslant 0$.

Conversely, if $u \in \mathcal{D}^{\prime}(\Omega)$ with $\Delta u \geqslant 0$, form $u_{\varepsilon}=u * \psi_{\varepsilon}$. Then $u_{\varepsilon} \in C^{\infty}\left(\Omega_{\varepsilon}\right)$ and $\Delta u_{\varepsilon} \geqslant 0$. Hence by Proposition 1.2.3, $u_{\varepsilon} \in S H\left(\Omega_{\varepsilon}\right)$. I claim that $u_{\varepsilon}$ is an increasing function of $\varepsilon$. To see this, note that the solution $\chi_{\varepsilon}$ of $\Delta \chi_{\varepsilon}=\psi_{\varepsilon}$ in $\mathbf{R}^{n} \backslash\{0\}$ which is zero for $|x|>\varepsilon$ can be written

$$
\chi_{\varepsilon}(x)=\int_{|x|}^{\varepsilon} s^{-n+1} d s \int_{s / \varepsilon}^{1} t^{n-1} \Psi(t) d t, \quad 0<|x| \leqslant \varepsilon,
$$

where $\Psi(|x|)=\psi(x)$. This formula shows that $\chi_{\varepsilon}$ is increasing in $\varepsilon>0$, because the integrand is non-negative and the domain of integration increases with $\varepsilon$. Now if $\varepsilon \geqslant \delta>0$, then $\chi_{\varepsilon}-\chi_{\delta} \in \mathcal{D}\left(\mathbf{R}^{n}\right)$ and $\psi_{\varepsilon}-\psi_{\delta}=\Delta\left(\chi_{\varepsilon}-\chi_{\delta}\right)$ in all of $\mathbf{R}^{n}$, not only in $\mathbf{R}^{n} \backslash\{0\}$. Moreover $\chi_{\varepsilon}-\chi_{\delta} \geqslant 0$, so that by the positivity of $\Delta u,\left(u *\left(\psi_{\varepsilon}-\psi_{\delta}\right)\right)(0)=u\left(\psi_{\varepsilon}-\psi_{\delta}\right) \geqslant 0$. Translating this we get $\left(u_{\varepsilon}-u_{\delta}\right)(x)=\left(u *\left(\psi_{\varepsilon}-\psi_{\delta}\right)\right)(x) \geqslant 0$ for all $x$ such that this has a sense, i.e., for all $x \in \Omega_{\varepsilon}$. This proves the claim that $u_{\varepsilon}$ is an increasing function of $\varepsilon$.

By known properties of subharmonic functions, the limit $f=\lim u_{\varepsilon}$ is subharmonic in $\Omega$, and since the convergence holds in $L_{l o c}^{1}(\Omega), f$ defines the distribution $u$. This proves the proposition.

If $f \in C^{2}(\Omega), \Omega \subset \mathbf{R}^{n}$, then by definition $f$ is convex if and only if the function $t \mapsto f(a+t b)=f_{a, b}(t)$ is convex for all $a \in \Omega$ and $b \in \mathbf{R}^{n}$ where it is defined. Hence by the chain rule

$$
f_{a, b}^{\prime \prime}(t)=\sum \frac{\partial^{2} f}{\partial x_{j} \partial x_{k}}(a+t b) b_{j} b_{k} \geqslant 0, \quad a, b \in \mathbf{R}^{n}, t \in \mathbf{R}, a+t b \in \Omega .
$$

It suffices to take $t=0$. We state the result as a proposition:

${ }^{2} \mathrm{~A}$ function is called radial if it is a function of the distance to the origin. 
Proposition 1.2.5. Let $\Omega \subset \mathbf{R}^{n}$ be convex and $f \in C^{2}(\Omega)$. Then $f$ is convex if and only if

$$
\sum \frac{\partial^{2} f}{\partial x_{j} \partial x_{k}}(a) b_{j} b_{k} \geqslant 0, \quad a \in \Omega, b \in \mathbf{R}^{n} .
$$

Proposition 1.2.6. Let $u \in \mathcal{D}^{\prime}(\Omega)$, where $\Omega \subset \mathbf{R}^{n}$ is convex. Then there exists $f \in C V X(\Omega)$ such that

$$
u(\varphi)=\int_{\Omega} f \varphi d \lambda, \quad \varphi \in \mathcal{D}(\Omega)
$$

if and only if

$$
\sum \frac{\partial^{2} u}{\partial x_{j} \partial x_{k}} b_{j} b_{k} \geqslant 0, \quad b \in \mathbf{R}^{n}
$$

in the sense of distributions.

Proof. If $f \in C V X(\Omega)$, form $f_{\varepsilon}=f * \psi_{\varepsilon} \in C V X\left(\Omega_{\varepsilon}\right)$ with $\psi$ as in the proof of Proposition 1.2.4. Then $f_{\varepsilon} \rightarrow f$ in $\mathcal{D}^{\prime}(\Omega)$, which implies

$$
\sum \frac{\partial^{2} f_{\varepsilon}}{\partial x_{j} \partial x_{k}} b_{j} b_{k} \rightarrow \sum \frac{\partial^{2} f}{\partial x_{j} \partial x_{k}} b_{j} b_{k}
$$

in $\mathcal{D}^{\prime}(\Omega)$, since convergence there is stable under differentiation. (We use here the weak topology $\sigma\left(\mathcal{D}^{\prime}(\Omega), \mathcal{D}(\Omega)\right)$, meaning that $u_{j} \rightarrow u$ if $u_{j}(\varphi) \rightarrow u(\varphi)$ for every test function $\varphi$.) Positivity is preserved under passage to the limit, which means that (1.2.4) holds.

Conversely, if $u$ satisfies the positivity condition (1.2.4), form $u_{\varepsilon}=u * \psi_{\varepsilon} \in C^{\infty}\left(\Omega_{\varepsilon}\right)$. Then also $u_{\varepsilon}$ satisfies the positivity condition (1.2.4), which is the same as (1.2.3) since $u_{\varepsilon}$ is a smooth function. Therefore $u_{\varepsilon}$ is convex by Proposition 1.2.5. Moreover $u_{\varepsilon}$ tends decreasingly (cf. the proof of Proposition 1.2.4) to some function $f$, which is then necessarily convex as a pointwise limit of convex functions. Since convergence holds in $L_{l o c}^{1}(\Omega), f$ defines the given distribution $u$.

Proposition 1.2.7. Let $f \in C^{2}(\Omega), \Omega \subset \mathbf{C}^{n}$. Then $f$ is plurisubharmonic if and only if

$$
\sum \frac{\partial^{2} f}{\partial z_{j} \partial \bar{z}_{k}}(a) b_{j} \bar{b}_{k} \geqslant 0, \quad a \in \Omega, b \in \mathbf{C}^{n} .
$$

Proof. This follows from the chain rule and Proposition 1.2.3.

Proposition 1.2.8. Let $u \in \mathcal{D}^{\prime}(\Omega), \Omega \subset \mathbf{C}^{n}$. Then there exists $f \in P S H(\Omega)$ such that $u(\varphi)=\int_{\Omega} f \varphi d \lambda$ for every test function $\varphi \in \mathcal{D}(\Omega)$ if and only if

$$
\sum \frac{\partial^{2} u}{\partial z_{j} \partial \bar{z}_{k}} b_{j} \bar{b}_{k} \geqslant 0, \quad b \in \mathbf{C}^{n}
$$

in the sense of distributions.

Proof. The proof is analogous to the convex case, Proposition 1.2.6.

It is now easy to prove the inclusions (1.1.5) and (1.1.6). The first follows from taking $b_{j}=\delta_{j}^{k}$ in (1.2.4) and then summing over $k$. In (1.1.6) the first inclusion follows from (1.1.5) and the second from (1.2.6): again take $b_{j}=\delta_{j}^{k}$ and sum over $k$. 
Proposition 1.2.9. Let $u \in P S H(\Omega)$ be locally independent of the imaginary part of $z$, i.e., for any $z \in \Omega, f\left(z^{\prime}\right)=f(z)$ if $z^{\prime}$ is sufficiently close to $z$ and $\operatorname{Re} z^{\prime}=\operatorname{Re} z$. Then $f$ is locally convex in $\Omega$ (thus convex if $\Omega$ is convex).

Proof. If $u$ is a plurisubharmonic function it satisfies (1.2.6), but if it is locally independent of the imaginary part of the variables $z_{j}$, that condition reduces to (1.2.4) for $u$ regarded as a function of the $x_{j}=\operatorname{Re} z_{j}$. Thus by Proposition 1.2.6 there is a locally convex function $f$ which defines the same distribution as $u$. The regularizations $u_{\varepsilon}$ and $f_{\varepsilon}$ are therefore equal, which implies that also their $\operatorname{limits}_{\lim _{\varepsilon \rightarrow 0}} u_{\varepsilon}=u$ and $\lim _{\varepsilon \rightarrow 0} f_{\varepsilon}=f$ are equal at every point.

Corollary 1.2.10. If $\Omega$ is a pseudoconvex open set in $\mathbf{C}^{n}$ which is independent of the imaginary parts of the variables in the sense that $z \in \Omega$ and $\operatorname{Re} z^{\prime}=\operatorname{Re} z$ implies $z \in \Omega$, then every component of $\Omega$ is convex.

Proof. Consider the function $u=-\log d$, where $d$ is the distance to the complement of $\Omega$. Thus $u$ is plurisubharmonic if $\Omega$ is pseudoconvex - this is indeed one of the possible definitions of pseudoconvexity; see Hörmander [1990:Theorem 2.6.7]. By the proposition, $u$ is locally convex. Therefore the restriction of $u$ to any segment contained in $\Omega$ is convex. Now if $a^{0}$ and $a^{1}$ are two points which belong to the same component of $\Omega$, there is a curve from one to the other, say $[0,1] \ni t \mapsto a^{t} \in \Omega$. We claim that the segment from $a^{0}$ to $a^{t}$ must be contained in $\Omega$ for all $t$. Indeed the set $T$ of all such $t$ is open in $[0,1]$ by the openness of $\Omega$, and it is closed by the definition of $u$, for the smallest distance from any point on the segment $\left[a^{0}, a^{t}\right]$ to the complement of $\Omega$ is never smaller than the distance from $\left\{a^{0}, a^{t}\right\}$ to $\mathbf{C}^{n} \backslash \Omega$ by the convexity of $u$ on $\left[a^{0}, a^{t}\right]$. Moreover $T$ is not empty, for $0 \in T$. This proves that $T$ is equal to all of $[0,1]$. Thus the segment $\left[a^{0}, a^{1}\right]$ is contained in $\Omega$.

These results illustrate some of the many analogies between the three cones $C V X, S H$ and $P S H$. Let us mention one aspect where this analogy is not clear. Given any cone $K$ in a vector space we may form the space $\delta K=K-K$ of all differences of elements of $K$. Thus we form three subspaces $\delta C V X(\Omega), \delta S H(\Omega)$ and $\delta P S H(\Omega)$ of $L_{l o c}^{1}(\Omega)$ (or $\mathcal{D}^{\prime}(\Omega)$ ) consisting of all differences of functions that are, respectively, convex and finite-valued, subharmonic and finite almost everywhere, and plurisubharmonic and finite almost everywhere in $\Omega(\Omega$ being convex and open in $\mathbf{R}^{n}$ in the first case, just open in the second, and open in $\mathbf{C}^{n}$ in the last case). Each of these spaces has a local variant consisting of those locally integrable functions that admit a representation $f=f_{1}-f_{2}$ with $f_{j} \in K$ in a neighborhood of an arbitrary point. It is now easy to prove that $\delta S H_{l o c}(\Omega)=\delta S H(\Omega)$ for all open sets (it is the space of all locally integrable functions $f$ such that $\Delta f$ is a measure). Also $\delta C V X_{l o c}(\Omega)=\delta C V X(\Omega)$ if $\Omega$ is convex. But it seems not to be known whether $\delta P S H_{l o c}(\Omega)=\delta P S H(\Omega)$ (for example in a pseudoconvex open set). See Kiselman [1977] for details.

\subsection{The minimum principle}

For any given function $f$ defined in $\mathbf{R}^{n} \times \mathbf{R}^{m}$, we call

$$
g(x)=\inf _{y \in \mathbf{R}^{m}} f(x, y), \quad x \in \mathbf{R}^{n},
$$

the marginal function of $f$. (It defines a kind of margin of the epigraph of $f$.) 
Theorem 1.3.1. Let $f: \mathbf{R}^{n} \times \mathbf{R}^{m} \rightarrow[-\infty,+\infty]$ be convex. Then its marginal function (1.3.1) is convex.

Proof. The strict epigraph of $f$ (cf. 1.1.4) is

$$
\operatorname{epi}_{s} f=\left\{(x, y, t) \in \mathbf{R}^{n} \times \mathbf{R}^{m} \times \mathbf{R} ; f(x, y)<t\right\} .
$$

We now observe that $\operatorname{epi}_{s} g=\pi\left(\operatorname{epi}_{s} f\right)$, where $\pi$ is the projection $(x, y, t) \mapsto(x, t)$. If $f$ is convex, then epi $f$ is convex, and any linear image of a convex set is convex, so epi ${ }_{s} g=$ $\pi\left(\operatorname{epi}_{s} f\right)$ is also convex. This means that the function $g$ is convex.

Calculus proof. (Not that it is necessary now-we shall do it only as a warm-up for the plurisubharmonic case.) Let us assume that the function is of class $C^{2}$ and that the infimum is attained at a point $y=w(x)$ for each $x$ which depends in a $C^{1}$ fashion on $x$ :

$$
y=\left(w_{1}(x), \ldots, w_{m}(x)\right)^{\mathrm{T}}
$$

where the exponent means transpose, so that $y$ is regarded as a column vector. Assume also $x \in \mathbf{R}$, i.e., $n=1$. This is enough; in general we consider $g\left(x^{0}+t x^{1}\right), t \in \mathbf{R}$.

Thus $g(x)=f(x, w(x))$; the chain rule yields

$$
\frac{\partial g}{\partial x}=\frac{\partial f}{\partial x}+\sum \frac{\partial f}{\partial y_{k}} \frac{\partial w_{k}}{\partial x}
$$

At a minimum point we have $\partial f / \partial y_{k}=0$, so that $\partial g / \partial x=\partial f / \partial x$ when $y=w(x)$. By the chain rule again

$$
\frac{\partial^{2} g}{\partial x^{2}}=\frac{\partial^{2} f}{\partial x^{2}}+\sum \frac{\partial^{2} f}{\partial x \partial y_{k}} \frac{\partial w_{k}}{\partial x}=f_{x x}+A \alpha
$$

where $A$ is the row matrix

$$
A=\left(A_{1}, \ldots, A_{m}\right) \text { with } A_{k}=\frac{\partial^{2} f}{\partial x \partial y_{k}}
$$

and $\alpha$ the column matrix

$$
\alpha=\left(\frac{\partial w_{1}}{\partial x}, \ldots, \frac{\partial w_{m}}{\partial x}\right)^{\mathrm{T}} .
$$

We now apply the chain rule to the equation $\partial f / \partial y_{k}(x, w(x))=0$, which gives

$$
\frac{\partial^{2} f}{\partial x \partial y_{k}}+\frac{\partial^{2} f}{\partial y_{j} \partial y_{k}} \frac{\partial w_{j}}{\partial x}=0, \text { in other words } A+\alpha^{\mathrm{T}} H=0
$$

where

$$
H=\left(\frac{\partial^{2} f}{\partial y_{j} \partial y_{k}}\right)
$$

is the Hessian matrix of $f$ with respect to $y$. Summing up:

$$
g_{x x}=f_{x x}+A \alpha=f_{x x}-\alpha^{\mathrm{T}} H \alpha .
$$


Now what do we know about $H$ ? The convexity of $f$ in all variables $\left(x, y_{1}, \ldots, y_{m}\right)$ implies that

$$
f_{x x}+\sum \frac{\partial^{2} f}{\partial x \partial y_{k}} b_{k}+\sum \frac{\partial^{2} f}{\partial y_{j} \partial x} b_{j}+\sum \frac{\partial^{2} f}{\partial y_{j} \partial y_{k}} b_{j} b_{k} \geqslant 0
$$

for all $b$ (column vectors) or

$$
f_{x x}+A b+b^{\mathrm{T}} A^{\mathrm{T}}+b^{\mathrm{T}} H b \geqslant 0 .
$$

Since $A b$ is a scalar, $A b=b^{\mathrm{T}} A^{\mathrm{T}}$ and we have $f_{x x}+2 A b+b^{\mathrm{T}} H b \geqslant 0$, and since $A=-\alpha^{\mathrm{T}} H$ this can be written as

$$
f_{x x}-2 \alpha^{\mathrm{T}} H b+b^{\mathrm{T}} H b \geqslant 0
$$

for any column vector $b$. Now choose $b=\alpha$. Then we finally obtain

$$
g_{x x}=f_{x x}-\alpha^{\mathrm{T}} H \alpha \geqslant 0
$$

and we are done.

During this calculation we needed that $w(x)$ is a $C^{1}$ function of $x$. It is the solution of the system $\partial f / \partial y_{j}=0$, and it follows from the implicit function theorem that $w$ is $C^{1}$ if the Hessian $H$ is positive definite, for the Hessian is precisely the Jacobian matrix of this system and we need the Jacobian (determinant) to be non-zero. Hence $w \in C^{1}$ and the chain rule can be applied as above. Note as a matter of curiosity that $g(x)=f(x, w(x))$ is $C^{1}$ since $w \in C^{1}$, but since $g_{x}=f_{x}$ when $y=w(x)$ we see that $g_{x}$ is also $C^{1}$, hence $g \in C^{2}$. This concludes our calculations on convex functions.

The condition that $f \in C^{2}$ and that the infimum is attained can be removed. Regularization and addition of a coercive function will help! We shall not show this now, since we shall do it soon in the plurisubharmonic case in detail.

We shall now investigate similarily the Levi form of a minimum of a plurisubharmonic function $f$. Thus as before $g(x)=f(x, w(x))$, where $y=w(x)$ defines a stationary point of $y \mapsto f(x, y)$. We let $x \in \mathbf{C}^{n}=\mathbf{C}$ and $y \in \mathbf{C}^{m}$. It is enough to consider $n=1$, because for plurisubharmonicity in $x$ we consider complex lines in $\mathbf{C}^{n}$.

We shall use the notation

$$
A_{k}=\frac{\partial^{2} f}{\partial x \partial y_{k}}, \quad B_{k}=\frac{\partial^{2} f}{\partial \bar{x} \partial y_{k}}, \quad H_{j k}=\frac{\partial^{2} f}{\partial y_{j} \partial y_{k}}, \quad L_{j k}=\frac{\partial^{2} f}{\partial y_{j} \partial \bar{y}_{k}},
$$

and put $A=\left(A_{1}, \ldots, A_{m}\right), B=\left(B_{1}, \ldots, B_{m}\right)$. Here $H=\left(H_{j k}\right)$ is the complex Hessian matrix and $L=\left(L_{j k}\right)$ is the Levi matrix with respect to the $y$ variables. We write

$$
\mathcal{H}(b)=\sum H_{j k} b_{j} b_{k}=b^{\mathrm{T}} H b
$$

for the Hessian form, which is a symmetric quadratic form (thus $H^{\mathrm{T}}=H, H^{*}=\bar{H}$ ), and

$$
\mathcal{L}(b)=\sum L_{j k} b_{j} \bar{b}_{k}=b^{\mathrm{T}} L \bar{b}
$$

for the Levi form, which is an Hermitian form if $f$ is real-valued; thus $L^{\mathrm{T}}=\bar{L}$ and $L^{*}=L$ in that case. We write $\alpha_{j}=\partial w_{j} / \partial x, \alpha=\left(\alpha_{1}, \ldots, \alpha_{m}\right)^{\mathrm{T}}$ and $\beta_{j}=\partial w_{j} / \partial \bar{x}, \beta=\left(\beta_{1}, \ldots, \beta_{m}\right)^{\mathrm{T}}$.

The result is this: 
Proposition 1.3.2. Let $f$ be a real-valued $C^{2}$ function in some open set $\Omega$ in the space of $1+m$ complex variables, $(x, y) \in \mathbf{C} \times \mathbf{C}^{m}$. If $y=w(x)$ is a stationary point of $y \mapsto f(x, y)$ which depends in a $C^{1}$ fashion on $x$, then the Laplacian of $g(x)=f(x, w(x))$ satisfies

$$
\frac{1}{4} \Delta g=g_{x \bar{x}}=f_{x \bar{x}}-2 \operatorname{Re} \mathcal{H}(\alpha, \beta)-\mathcal{L}(\alpha)-\mathcal{L}(\beta),
$$

where $\mathcal{H}$ and $\mathcal{L}$ are given by (1.3.2-4) and $\mathcal{H}(\alpha, \beta)=\alpha^{\mathrm{T}} H \beta$ is obtained by polarization.

Proof. If we differentiate $g(x)=f(x, w(x))$ once we get

$$
g_{x}(x)=\frac{\partial g}{\partial x}(x)=f_{x}(x, w(x))+\sum \frac{\partial f}{\partial y_{j}} \alpha_{j}+\sum \frac{\partial f}{\partial \bar{y}_{j}} \beta_{j}=f_{x}(x, w(x)),
$$

since $\partial f / \partial y_{j}$ and $\partial f / \partial \bar{y}_{j}$ both vanish at a stationary point. This shows that $g_{x}$ is of class $C^{1}$. We can therefore apply $\partial / \partial \bar{x}$ to the equation $g_{x}=f_{x}$ and get

$$
g_{x \bar{x}}=\frac{\partial^{2} g}{\partial x \partial \bar{x}}=\frac{\partial^{2} f}{\partial x \partial \bar{x}}+\sum \frac{\partial^{2} f}{\partial x \partial y_{k}} \frac{\partial w_{k}}{\partial \bar{x}}+\sum \frac{\partial^{2} f}{\partial x \partial \bar{y}_{k}} \frac{\partial \bar{w}_{k}}{\partial \bar{x}} .
$$

Since $f$ is real-valued it follows that $\partial^{2} f / \partial x \partial \bar{y}_{k}=\bar{B}_{k}$, thus

$$
g_{x \bar{x}}=f_{x \bar{x}}+\sum A_{k} \beta_{k}+\sum \bar{B}_{k} \bar{\alpha}_{k}=f_{x \bar{x}}+A \beta+\overline{B \alpha} .
$$

To determine $A$ and $B$ we differentiate the equation $\partial f / \partial y_{k}=0$ with respect to $x$ to get

$$
\frac{\partial^{2} f}{\partial x \partial y_{k}}+\sum \frac{\partial^{2} f}{\partial y_{j} \partial y_{k}} \frac{\partial w_{j}}{\partial x}+\sum \frac{\partial^{2} f}{\partial \bar{y}_{j} \partial y_{k}} \frac{\partial \bar{w}_{j}}{\partial x}=0
$$

or $A_{k}+\sum \alpha_{j} H_{j k}+\sum \bar{\beta}_{j} L_{j k}^{\mathrm{T}}=0$, which in matrix notation becomes

$$
A=-\alpha^{\mathrm{T}} H-\beta^{*} L^{\mathrm{T}} .
$$

Next we differentiate $\partial f / \partial y_{k}=0$ with respect to $\bar{x}$ and get

$$
\frac{\partial^{2} f}{\partial \bar{x} \partial y_{k}}+\sum \frac{\partial^{2} f}{\partial y_{j} \partial y_{k}} \frac{\partial w_{j}}{\partial \bar{x}}+\sum \frac{\partial^{2} f}{\partial \bar{y}_{j} \partial y_{k}} \frac{\partial \bar{w}_{j}}{\partial \bar{x}}=0
$$

or $B_{k}+\sum \beta_{j} H_{j k}+\sum \bar{\alpha}_{j} L_{k j}=0$, which in matrix notation gives

$$
B=-\beta^{\mathrm{T}} H-\alpha^{*} L^{\mathrm{T}} .
$$

Now insert the values $A=-\alpha^{\mathrm{T}} H-\beta^{*} L^{\mathrm{T}}$ and $B=-\beta^{\mathrm{T}} H-\alpha^{*} L^{\mathrm{T}}$ into (1.3.6). Then we get

$$
g_{x \bar{x}}=f_{x \bar{x}}-\alpha^{\mathrm{T}} H \beta-\beta^{*} L^{\mathrm{T}} \beta-\overline{\beta^{\mathrm{T}} H \alpha}-\overline{\alpha^{*} L^{\mathrm{T}} \alpha}=f_{x \bar{x}}-2 \operatorname{Re}\left(\alpha^{\mathrm{T}} H \beta\right)-\alpha^{\mathrm{T}} L \bar{\alpha}-\beta^{\mathrm{T}} L \bar{\beta},
$$

which in terms of $\mathcal{H}$ and $\mathcal{L}$ is just (1.3.5). This proves Proposition 1.3.2.

So far we have not assumed any plurisubharmonicity! We have just used the identity $g(x)=f(x, w(x))$, where $\partial f / \partial y_{k}(x, w(x))=0$ and $\partial f / \partial \bar{y}_{k}(x, w(x))=0$, equations which hold since $y=w(x)$ is a stationary point of $y \mapsto f(x, y)$. Note, by the way, that these two equations are equivalent if $f$ is real-valued. We shall now assume that $f$ is plurisubharmonic, and deduce a lower bound for its partial Laplacian with respect to $x$ : 
Proposition 1.3.3. If $f$ is plurisubharmonic and of class $C^{2}$ in an open set in $\mathbf{C} \times \mathbf{C}^{m}$, then

$$
f_{x \bar{x}}=\frac{\partial^{2} f}{\partial x \partial \bar{x}} \geqslant \bar{B} M B^{\mathrm{T}}
$$

where

$$
B=\left(B_{1}, \ldots, B_{m}\right), B_{k}=\frac{\partial^{2} f}{\partial \bar{x} \partial y_{k}},
$$

and $M$ is an Hermitian quasi-inverse of the Levi matrix

$$
L=\left(\frac{\partial^{2} f}{\partial y_{j} \partial \bar{y}_{k}}\right)
$$

i.e., $M^{*}=M$ and $L M L=L$.

Remark. In a nice coordinate system $L=L_{1} \oplus 0$, where $L_{1}$ is positive definite. Any Hermitian quasi-inverse then has the form $M=M_{1} \oplus M_{2}=L_{1}^{-1} \oplus M_{2}$, where $M_{2}$ is Hermitian. We get $L M=M L=I \oplus 0$, so $L M L=L$. Moreover $M L M=L_{1}^{-1} \oplus 0\left(=M\right.$ if $\left.M_{2}=0\right)$.

Proof of Proposition 1.3.3. What does it mean that $f$ is plurisubharmonic? By Proposition 1.2.7 it means that

$$
\frac{\partial^{2} f}{\partial x \partial \bar{x}} s \bar{s}+\sum \frac{\partial^{2} f}{\partial x \partial \bar{y}_{k}} s \bar{z}_{k}+\sum \frac{\partial^{2} f}{\partial y_{j} \partial \bar{x}} z_{j} \bar{s}+\sum \frac{\partial^{2} f}{\partial y_{j} \partial \bar{y}_{k}} z_{j} \bar{z}_{k} \geqslant 0,
$$

for all $s \in \mathbf{C}, z \in \mathbf{C}^{m}$. It suffices to take $s=1$ :

$$
f_{x \bar{x}}+\overline{B z}+B z+z^{\mathrm{T}} L \bar{z} \geqslant 0, \quad z \in \mathbf{C}^{n},
$$

or equivalently

$$
f_{x \bar{x}} \geqslant-\inf _{z}\left(z^{\mathrm{T}} L \bar{z}+B z+\overline{B z}\right) .
$$

To find the best possible use of the plurisubharmonicity we need to determine the infimum in terms of $B$. The result is this:

Lemma 1.3.4. Suppose $F(z)=z^{\mathrm{T}} L \bar{z}+2 \operatorname{Re} B z$ is bounded from below. Then its infimum is

$$
\inf _{z \in \mathbf{C}^{m}}\left(z^{\mathrm{T}} L \bar{z}+2 \operatorname{Re} B z\right)=-\bar{B} M B^{\mathrm{T}}
$$

and is attained at $z=-\bar{M} B^{*}$, where $M$ is any Hermitian quasi-inverse of $L$. (Then $L M B^{\mathrm{T}}=$ $B^{\mathrm{T}}$, and this property is sufficient for the formula above to hold.)

Proof of Lemma 1.3.4. If the infimum is attained at a point $a$, we must have

$$
F(z)=(z-a)^{\mathrm{T}} \overline{L(z-a)}-a^{\mathrm{T}} L \bar{a}
$$

for the linear terms must vanish in an expansion around $a$. Hence $\inf F(z)=-a^{\mathrm{T}} L \bar{a}$. Now assume $M$ is such that $L M B^{\mathrm{T}}=B^{\mathrm{T}}$ and $M^{*}=M$. Then we just calculate:

$$
F(z)=z^{\mathrm{T}} L \bar{z}+2 \operatorname{Re} B z=\left(z+\bar{M} B^{*}\right)^{\mathrm{T}} L \overline{\left(z+\bar{M} B^{*}\right)}-\bar{B} M L M B^{\mathrm{T}}
$$


Thus inf $F=-\bar{B} M L M B^{\mathrm{T}}=-\bar{B} M B^{\mathrm{T}}$ and it is attained at the point $z=-\bar{M} B^{*}$ (not necessarily unique, since it depends on the choice of quasi-inverse). Here we only used the fact that $M$ satisfies $L M B^{\mathrm{T}}=B^{\mathrm{T}}$ and $M^{*}=M$.

For completeness we shall also show that if $L M L=L, M^{*}=M$, then necessarily $L M B^{\mathrm{T}}=B^{\mathrm{T}}$. If this is not true there is a row-vector $c$ such that $c B^{\mathrm{T}} \neq 0$ but $c L=0$. (We have $L M x=x$ for all columns of $L$, hence for $x$ in the linear span of those columns, so if $B^{\mathrm{T}}$ does not belong to this span, there is a linear form which annihilates the columns of $L$ without annihilating $B^{\mathrm{T}}$ ). Now consider

$$
F\left(s c^{\mathrm{T}}\right)=\left(s c^{\mathrm{T}}\right)^{\mathrm{T}} \overline{L\left(s c^{\mathrm{T}}\right)}+B s c^{\mathrm{T}}+\overline{B s c^{\mathrm{T}}}=2 \operatorname{Re}\left(s B c^{\mathrm{T}}\right) .
$$

This real-linear form is not identically zero by hypothesis, and hence not bounded from below. But we assumed $F$ to be bounded from below. The set of all column vectors $x$ such that $L M x=x$ includes all columns of $L$ and therefore also $B^{\mathrm{T}}$.

Thus Lemma 1.3.4 and hence Proposition 1.3.3 are proved.

Theorem 1.3.5. Let $f$ be plurisubharmonic and of class $C^{2}$ in an open set in $\mathbf{C} \times \mathbf{C}^{m}$ and $y=w(x)$ a stationary point of $y \mapsto f(x, y)$ with $w$ of class $C^{1}$. We write

$$
\beta=\left(\frac{\partial w_{1}}{\partial \bar{x}}, \ldots, \frac{\partial w_{m}}{\partial \bar{x}}\right)^{\mathrm{T}}, \quad H=\left(\frac{\partial^{2} f}{\partial y_{j} \partial y_{k}}\right), \quad L=\left(\frac{\partial^{2} f}{\partial y_{j} \partial \bar{y}_{k}}\right)
$$

and let $M$ be an arbitrary Hermitian quasi-inverse of L, i.e., $M=M^{*}, L M L=L$. Define

$$
N=H M^{\mathrm{T}} \bar{H}-L .
$$

Then $g(x)=f(x, w(x))$ satisfies

$$
g_{x \bar{x}} \geqslant \beta^{\mathrm{T}}\left(H M^{\mathrm{T}} \bar{H}-L\right) \bar{\beta}=\mathcal{M}(\overline{H \beta})-\mathcal{L}(\beta)=\beta^{\mathrm{T}} N \bar{\beta}=\mathcal{N}(\beta),
$$

where $\mathcal{M}(b)=b^{\mathrm{T}} M \bar{b}$ and $\mathcal{N}(b)=b^{\mathrm{T}} N \bar{b}$ denote the Hermitian forms defined by $M$ and $N$ (cf. (1.3.4)). In particular, $g$ is subharmonic if $\mathcal{N}(\beta) \geqslant 0$.

Thus for every plurisubharmonic function $f$ of class $C^{2}$ we have defined an Hermitian matrix $N=H M^{\mathrm{T}} \bar{H}-L$ which is of interest. It is highly non-linear in $f$.

Proof. The criterion (1.3.9) of Proposition 1.3.3, $f_{x \bar{x}} \geqslant \bar{B} M B^{\mathrm{T}}$, takes the form $f_{x \bar{x}} \geqslant$ $\mathcal{M}(\overline{H \beta})+2 \operatorname{Re} \mathcal{H}(\alpha, \beta)+\mathcal{L}(\alpha)$ if we are at a critical point. Indeed, $B=-\beta^{\mathrm{T}} H-\alpha^{*} L^{\mathrm{T}}$ (see $(1.3 .8))$, so

$$
\bar{B} M B^{\mathrm{T}}=\beta^{*} \bar{H} M H \beta+\beta^{*} \bar{H} M L \bar{\alpha}+\alpha^{\mathrm{T}} L M H \beta+\alpha^{\mathrm{T}} L M L \bar{\alpha} .
$$

To simplify this expression we use the equations $L M L=L$ and $L M B^{\mathrm{T}}=B^{\mathrm{T}}$, which give $L M H \beta=H \beta$ and $\beta^{*} \bar{H} M L=\beta^{*} \bar{H}$. Therefore

$$
f_{x \bar{x}} \geqslant \bar{B} M B^{\mathrm{T}}=\beta^{*} \bar{H} M H \beta+\beta^{*} \bar{H} \bar{\alpha}+\alpha^{\mathrm{T}} H \beta+\alpha^{\mathrm{T}} L \bar{\alpha}=\mathcal{M}(\overline{H \beta})+2 \operatorname{Re} \mathcal{H}(\alpha, \beta)+\mathcal{L}(\alpha) .
$$

On the other hand we calculated $g_{x \bar{x}}$ in Proposition 1.3.2:

$$
g_{x \bar{x}}=f_{x \bar{x}}-2 \operatorname{Re} \mathcal{H}(\alpha, \beta)-\mathcal{L}(\alpha)-\mathcal{L}(\beta) .
$$


Using the estimate for $f_{x \bar{x}}$ we get $g_{x \bar{x}} \geqslant \mathcal{M}(\overline{H \beta})-\mathcal{L}(\beta)=\mathcal{N}(\beta)$, which concludes the proof of the theorem.

Let us look at a few special cases of the theorem.

1. If $w$ is a holomorphic function, then $\beta=0$ so $g$ is subharmonic. This is no surprise, $g(x)=$ $f(x, w(x))$ being the composition of a plurisubharmonic function and a holomorphic mapping.

2. If $N=H M^{\mathrm{T}} \bar{H}-L \geqslant 0$ (positive semi-definite), then $g$ is subharmonic.

3. The term $\beta^{\mathrm{T}} H M^{\mathrm{T}} \overline{H \beta}$ is equal to $x^{\mathrm{T}} M^{\mathrm{T}} \bar{x}$ with $x=H \beta$, so it is always greater than or equal to zero if $L \geqslant 0$. Therefore $g_{x \bar{x}} \geqslant-\mathcal{L}(\beta)$. Suppose we know that $L \leqslant a I,|\beta| \leqslant b$. Then $-\beta L \beta^{*} \geqslant-a|\beta|^{2} \geqslant-a b^{2}$, so that $g(x)+a b^{2}|x|^{2}$ is subharmonic. This means that we have some control of the lack of subharmonicity.

4. If $L$ is invertible, the condition $H M^{\mathrm{T}} \bar{H} \geqslant L$ means that $P=L^{-1} H$ satisfies $P \bar{P} \geqslant I$. Is there a nice interpretation of this inequality?

5. For $m=1$ it is easy to analyze the condition. It becomes

$$
g_{x \bar{x}} \geqslant(H M \bar{H}-L)|\beta|^{2} .
$$

Hence $g$ is subharmonic if either $\beta=0$ or $|H| \geqslant L$. At a minimum we must have $|H| \leqslant L$, so the case $|H| \geqslant L$ is then equivalent to $|H|=L$, which means that there exists a direction where the second derivative is zero. (If $m>1$ and $L$ and $H$ can be diagonalized simultaneously then we have more or less this case.)

6. Again for $m=1$, the expression $N=H M \bar{H}-L$ is equal to

$$
N=\frac{f_{y^{\prime} y^{\prime \prime}}^{2}-f_{y^{\prime} y^{\prime}} f_{y^{\prime \prime} y^{\prime \prime}}}{f_{y^{\prime} y^{\prime}}+f_{y^{\prime \prime} y^{\prime \prime}}}=-\frac{\text { real Monge-Ampère }(f)}{\text { Laplacian }(f)},
$$

where $y=y^{\prime}+i y^{\prime \prime}, y^{\prime}, y^{\prime \prime} \in \mathbf{R}$. Same conclusion as in 5 .

7. Consider the special case $L=0$. Then $f$ is plurisubharmonic if $B=0$ and $f_{x \bar{x}} \geqslant 0$. Taking $M=0$ in the theorem we see that $g_{x \bar{x}} \geqslant 0$, which is true, since in Proposition 1.3.2 we have $g_{x \bar{x}}=f_{x \bar{x}}$. Indeed, $0=B=-\beta^{\mathrm{T}} H$, so $\mathcal{H}(\alpha, \beta)=\alpha^{\mathrm{T}} H \beta=0$. The conclusion cannot be improved.

8. Consider now the special case $H=0$. Then $f$ is plurisubharmonic if and only if $f_{x \bar{x}} \geqslant \mathcal{L}(\alpha)$. In fact, the necessary and sufficient condition for plurisubharmonicity (see $(1.3 .10))$ is

$f_{x \bar{x}} \geqslant-\inf _{z}(\mathcal{L}(z)+2 \operatorname{Re} B z)=-\inf _{z}\left(\mathcal{L}(z)-2 \operatorname{Re} \alpha^{*} L^{\mathrm{T}} z\right)=-\inf _{z}(\mathcal{L}(\alpha-z)-\mathcal{L}(\alpha))=\mathcal{L}(\alpha)$.

In Proposition 1.3.2 we have $g_{x \bar{x}}=f_{x \bar{x}}-\mathcal{L}(\alpha)-\mathcal{L}(\beta)$. The theorem says that $g_{x \bar{x}} \geqslant$ $-\mathcal{L}(\beta)$, which is true and cannot be improved.

We have thus seen in 7 . and 8 . that if either $H$ or $L$ vanishes, the conclusion of the theorem cannot be improved.

9. If $f$ is independent of $\operatorname{Im} y$, then $H=L$, so

$$
N=H M^{\mathrm{T}} \bar{H}-L=L M^{\mathrm{T}} \bar{L}-L=(L M L)^{\mathrm{T}}-L^{\mathrm{T}}=0^{\mathrm{T}}=0,
$$

for $L^{*}=L, H^{\mathrm{T}}=H$. So then the matrix $N$ vanishes identically! Thus we have proved: 
Corollary 1.3.6. If $f \in C^{2}(\Omega) \cap P S H(\Omega)$ is locally independent of $\operatorname{Im} y$, then $g(x)=$ $f(x, w(x))$ is plurisubharmonic if $y=w(x)$ is a stationary point (local minimum) of the function $y \mapsto f(x, y)$ which depends in a $C^{1}$ manner of $x$.

It is now a matter of routine to eliminate the smoothness assumptions in Corollary 1.3.6. We then obtain the following theorem:

Theorem 1.3.7 (The Minimum Principle, Kiselman [1978]). Let $\Omega \subset \mathbf{C}^{n} \times \mathbf{C}^{m}$ be pseudoconvex and $f \in P S H(\Omega)$. Assume that $\Omega$ and $f$ are both independent of the imaginary part of $y \in \mathbf{C}^{m}$, i.e., if $(x, y) \in \Omega$ and $y^{\prime}$ is a point in $\mathbf{C}^{m}$ with $\operatorname{Re} y_{j}^{\prime}=\operatorname{Re} y_{j}$, then $\left(x, y^{\prime}\right) \in \Omega$ and $f\left(x, y^{\prime}\right)=f(x, y)$. Assume also (now only for simplicity) that the fiber $\pi^{-1}(x) \cap \Omega$ is connected (thus a convex set according to Corollary 1.2.10) for each $x \in \mathbf{C}^{n}$, where $\pi$ is the projection $\mathbf{C}^{n} \times \mathbf{C}^{m} \rightarrow \mathbf{C}^{n}$ defined by $\pi(x, y)=x$. Define

$$
g(x)=\inf _{y} f(x, y) .
$$

Then $\pi(\Omega)$ is pseudoconvex and $g \in P S H(\pi(\Omega))$.

Remarks. If the fiber $\pi^{-1}(x)$ is not connected, it consists of several convex components, and the theorem makes sense in this case also; however, the function $g$ will not be defined in a subset of $\mathbf{C}^{n}$ but on a Riemann domain over $\mathbf{C}^{n}$. See Kiselman [1978] for details. - If $m=1$, then each component of a fiber $\pi^{-1}(x) \cap \Omega$ is a strip or a half-plane or the whole plane. In most of the applications that we are going to present we do have $m=1$, and the fiber is a half-plane, in particular connected.

A special case of the theorem is when $f=0$ in $\Omega$ and $g=0$ in $\pi(\Omega)$. Then the theorem just says that the projection $\pi(\Omega)$ is pseudoconvex. This special case is equivalent to the whole theorem. Indeed, let

$$
\Omega_{f}=\{(x, y, t) \in \Omega \times \mathbf{C} ; f(x, y)<\operatorname{Re} t\} .
$$

Then

$$
\pi\left(\Omega_{f}\right)=\{(x, t) \in \pi(\Omega) \times \mathbf{C} ; g(x)<\operatorname{Re} t\} .
$$

It is known that $\Omega_{f}$ is pseudoconvex if and only if $\Omega$ is pseudoconvex and $f \in P S H(\Omega)$. Therefore, if we have proved the theorem in the special case of zero functions, it follows that $\pi\left(\Omega_{f}\right)$ is pseudoconvex, which is equivalent to $g$ being plurisubharmonic.

Proof of Theorem 1.3.7. We shall successively reduce the theorem to Corollary 1.3.6.

First we shall show that if the result holds for a function $f$ which tends to $+\infty$ at the boundary in the sense that the set

$$
\Omega^{a}=\{(x, y) \in \Omega ; f(x, y)<a\}
$$

satisfies

$$
\Omega^{a} \cap\left(\mathbf{C}^{n} \times \mathbf{R}^{m}\right) \Subset \Omega, \quad a \in \mathbf{R},
$$

then it holds generally. To do this we form

$$
f_{j}=\max (-j, f)+\frac{1}{j}\left(\max (0,-\log d)+|x|^{2}+|\operatorname{Re} y|^{2}\right),
$$


where $d$ is the distance to the complement of $\Omega$. The functions $f_{j}$ satisfy (1.3.13), and if the result holds for them, so that $g_{j}=\inf _{y} f_{j}(x, y)$ is plurisubharmonic, then it follows that $\lim g_{j}=\inf _{j} g_{j}$ is plurisubharmonic. Clearly the decreasing limit $\inf _{j} g_{j}$ is precisely $g$. This means that the theorem holds for general $f$.

Next suppose that a function $f$ satisfies (1.3.13). Then we form

$$
f_{\varepsilon}=f * \psi_{\varepsilon}+\varepsilon|\operatorname{Re} y|^{2}
$$

like in the proof of Proposition 1.2.4, but of course with $\psi_{\varepsilon}(x, y)=\varepsilon^{-n-m} \psi((x, y) / \varepsilon)$. This convolution is well-defined in the set $\Omega_{\varepsilon}$ of points of distance larger than $\varepsilon$ to the complement of $\Omega$. Given an arbitrary relatively compact subdomain $\omega$ of $\pi(\Omega)$ we shall prove that $g$ is plurisubharmonic in $\omega$. Now $g$ is bounded from above in $\omega$, say $g<a$ there. Pick $\varepsilon$ with $0<\varepsilon \leqslant 1$ and $b>a$ such that $\Omega^{a}+\varepsilon B \subset \Omega^{b}$. Then $\Omega_{\varepsilon}$ contains $\Omega^{a}$, so that $f * \psi_{e}$ is well-defined in $\Omega^{a}$. Next let

$$
c=b+\sup _{(x, y) \in \Omega^{a}}|\operatorname{Re} y|^{2}<+\infty .
$$

Then $\Omega^{a} \subset \Omega^{c} \subset \Omega_{\delta}$ for some small positive $\delta$. For $x \in \omega$ we have

$$
c>\inf _{y}\left(f_{\varepsilon}(x, y) ;(x, y) \in \Omega^{a}\right) \geqslant \inf _{y}\left(f_{\varepsilon}(x, y) ;(x, y) \in \Omega^{c}\right) .
$$

In $\Omega_{\varepsilon} \backslash \Omega^{c}$ we have $f_{\varepsilon} \geqslant f \geqslant c$, so the last infinimum is equal to $\inf _{y}\left(f_{\varepsilon}(x, y) ;(x, y) \in \Omega_{\varepsilon}\right)$; we denote this quantity by $g_{\varepsilon}(x)$.

Thus $f_{\varepsilon}$ is a strongly convex ${ }^{3}$ function of Re $y$ and the infimum when $y$ varies is attained at a unique real point $y=w_{\varepsilon}(x)$. Corollary 1.3 .6 can be applied to such functions. To see this, we first have to prove that the function $w_{\varepsilon}$ is well-defined and of class $C^{1}$. Now this follows from the implicit function theorem, for the point $y$ is the solution of the system of equations $\partial f_{\varepsilon} / \partial y_{j}=0$, whose Jacobian is

$$
\operatorname{det}_{j, k}\left(\frac{\partial^{2} f_{\varepsilon}}{\partial\left(\operatorname{Re} y_{j}\right) \partial\left(\operatorname{Re} y_{k}\right)}\right)(x, w(x))
$$

But this determinant is also the determinant of the real Hessian matrix of $f_{\varepsilon}$ as a function of $\operatorname{Re} y$, and is therefore non-zero in view of the strong convexity of $f_{\varepsilon}$ as a function of $\operatorname{Re} y$. This proves that $w_{\varepsilon}$ is of class $C^{\infty}$.

We also have to ensure that the fibers $\pi^{-1}(x) \cap \Omega_{\varepsilon}$ are connected, even though the set $\Omega_{\varepsilon}$ itself need not be connected. To see this, define first

$$
W_{x}(\varepsilon)=\left\{y \in \mathbf{C}^{m} ;(x, y)+\left(\varepsilon B \cap\left(\{0\} \times \mathbf{C}^{m}\right)\right) \subset \Omega\right\} \subset \mathbf{C}^{m}, \quad x \in \mathbf{C}^{n}, \varepsilon>0 .
$$

Since $\pi^{-1}(x) \cap \Omega$ is connected, thus convex, the set $W_{x}(\varepsilon)$ is convex. Therefore $\{x\} \times W_{x^{\prime}}(\varepsilon)$ is convex as well; it is a subset of $\pi^{-1}(x)$. But then also the intersection

$$
\bigcap_{x^{\prime} \in x+\varepsilon B}\{x\} \times W_{x^{\prime}}\left(\sqrt{\varepsilon^{2}-\left|x^{\prime}-x\right|^{2}}\right)=\pi^{-1}(x) \cap \Omega_{\varepsilon}
$$

is convex. Thus Corollary 1.3.6 can be applied, and we deduce that $g_{\varepsilon}(x)=\inf _{y} f_{\varepsilon}(x, y)$ is a plurisubharmonic function of $x$. Letting $\varepsilon$ tend to 0 , we conclude that $g=\lim _{\varepsilon \rightarrow 0} g_{\varepsilon}=\inf _{\varepsilon} g_{\varepsilon}$ is plurisubharmonic in $\omega$. Since $\omega$ was an arbitrary relatively compact subdomain of $\pi(\Omega)$, this proves the theorem in general.

${ }^{3}$ This means that we can subtract a small positive multiple of $|\operatorname{Re} y|^{2}$ and still have a convex function. 


\section{Chapter 2. The Lelong number and the integrability index}

\subsection{Introduction}

In the present chapter we shall show how to construct in a straight-forward way new plurisubharmonic functions from old ones using standard methods of convex analysis. These new functions can then be used to find analytic varieties that are connected with the original function, or rather with its singularities. We shall therefore first describe how one can measure the singularity of a plurisubharmonic function: this is done using the Lelong number and the integrability index.

The Lelong number measures how big (or "heavy") the singularities of a plurisubharmonic function are. It generalizes the notion of multiplicity of a zero of a holomorphic function. To define it, we first form the measure $\mu=(2 \pi)^{-1} \Delta f$, where $\Delta$ is the Laplacian in all $2 n$ real variables $\operatorname{Re} z_{j}, \operatorname{Im} z_{j}$. Note that when $f=\log |h|$ is the logarithm of the absolute value of a holomorphic function of one variable, then $\mu$ is a sum of point masses, one at each zero of $h$ and with weight equal to the multiplicity of the zero. The Lelong number of $f$ at a point $x$ is by definition the $(2 n-2)$-dimensional density of the measure $\mu$ at $x$. More explicitly, it is the limit as $r \rightarrow 0$ of the mean density of $\mu$ in the ball of center $x$ and radius $r$ :

$$
\nu_{f}(x)=\lim _{r \rightarrow 0} \frac{\mu(x+r B)}{\lambda_{2 n-2}\left(r B \cap \mathbf{C}^{n-1}\right)},
$$

where $\lambda_{k}$ denotes $k$-dimensional Lebesgue measure. Note that we compare the mass of $\mu$ in the ball $x+r B$ with the volume of the ball of radius $r$ in $\mathbf{C}^{n-1}$, i.e., of real dimension $2 n-2$. This makes sense, because if $f=\log |h|$ with $h$ holomorphic, then $\mu$ is a mass distribution on the $(2 n-2)$-dimensional zero set of $h$. If $n=1$, then $\lambda_{2 n-2}\left(r B \cap \mathbf{C}^{n-1}\right)=\lambda_{0}(\{0\})=1$, and $\nu_{f}(x)$ is just the mass of $\mu$ at $x$.

One often approximates a plurisubharmonic function $f$ by $f_{j}=\max (-j, f)$ or by smooth functions $f_{j}=f * \psi_{j}$ obtained by convolution. However, in these cases the functions $f_{j}$ never take the value $-\infty$, so their Lelong numbers $\nu_{f_{j}}(x)$ are zero everywhere; their singularities as measured by the Lelong number do not approach those of $f$ as $j \rightarrow+\infty$. Here we shall construct functions $f_{\tau}$ depending on a non-negative number $\tau$ such that $f_{0}=f$ and $f_{\tau}$ has Lelong number $\nu_{f_{\tau}}(x)=\left(\nu_{f}(x)-\tau\right)^{+}$. It turns out that the family $\left(f_{\tau}\right)_{\tau}$ can be used in various constructions. The singularities of $f_{\tau}$ are the same as those of $f$ but attenuated in a certain sense. More precisely, the important property is that $\nu_{f_{\tau}}(x)>0$ if $\tau<\nu_{f}(x)$, whereas the singularity is completely killed, i.e., $\nu_{f_{\tau}}(x)=0$, if $\tau>\nu_{f}(x)$. In this context it is convenient to define the Lelong number of a family of plurisubharmonic functions. We prove analyticity theorems for the superlevel sets of such numbers; see section 2.4.

If $f$ is plurisubharmonic and $t$ a positive number, the function $\exp (-f / t)$ may or may not be integrable. The set of all $t$ such that this function is locally integrable in the neighborhood of a certain point is an interval, and its endpoint measures how singular $f$ is. This is the 
reason behind the integrability index $\iota_{f}$ to be defined in section 2.3 (see (2.3.4)). From the Hörmander-Bombieri theorem we get analyticity theorems for the integrability index (see (2.3.4)). There is a relation between the integrability index and the Lelong number: $\iota_{f} \leqslant \nu_{f} \leqslant n \iota_{f}$, where $n$ is the complex dimension of the space; see Theorem 2.3.5. This relation cannot be improved (see Example 2.3.6), but nevertheless it will suffice to yield analyticity theorems for the Lelong number. The reason for this is roughly speaking that if we subtract the same quantity $\tau$ from two numbers like $\nu_{f}(x)$ and $\nu_{f}\left(x^{\prime}\right)>\nu_{f}(x)>\tau$, then the quotient between $\nu_{f}\left(x^{\prime}\right)-\tau$ and $\nu_{f}(x)-\tau$ can be large, for instance larger than the dimension $n$. This is why analyticity theorems for sets of plurisubharmonic functions are useful when it comes to proving analyticity theorems for a single function. For other studies of Lelong numbers, see Abrahamsson [1988], Demailly [1987, 1989], and Wang [1991].

\subsection{Spherical means and spherical suprema}

Let $f$ and $q$ be two given plurisubharmonic functions in an open set $\Omega$ in $\mathbf{C}^{n}$, thus $f, q \in$ $P S H(\Omega)$. We define an open set $\Omega_{q}$ in $\mathbf{C}^{n} \times \mathbf{C}$ as

$$
\Omega_{q}=\{(x, t) \in \Omega \times \mathbf{C} ; q(x)+\operatorname{Re} t<0\}
$$

and we note immediately that $\Omega_{q}$ is pseudoconvex if $\Omega$ is pseudoconvex, for the function $(x, t) \mapsto q(x)+\operatorname{Re} t$ is plurisubharmonic in $\Omega \times \mathbf{C}$. We shall assume that $q(x) \geqslant-\log d_{\Omega}(x)$ for all $x \in \Omega$, denoting by $d_{\Omega}(x)$ the distance from $x$ to the complement of $\Omega$, and we note that then $(x, t) \in \Omega_{q}$ implies that the closed ball of center $x$ and radius $\left|e^{t}\right|$ is contained in $\Omega$. We define two functions $u$ and $U$ in $\Omega_{q}$ by putting

$$
\begin{gathered}
u(x, t)=u_{f}(x, t)=u_{f, q}(x, t)=f_{z \in S} f\left(x+e^{t} z\right), \quad(x, t) \in \Omega_{q} ; \\
U(x, t)=U_{f}(x, t)=U_{f, q}(x, t)=\sup _{z \in S} f\left(x+e^{t} z\right), \quad(x, t) \in \Omega_{q} .
\end{gathered}
$$

Here $S$ is the Euclidean unit sphere, and the barred integral sign indicates the mean value; see (1.2.2). So $u_{f}(x, t)$ is the mean value of $f$ over the sphere $x+e^{t} S$, and $U_{f}(x, t)$ is the supremum of $f$ over the same sphere. Since we usually keep $q$ fixed, the dependence on that function need not always be shown. If $\Omega \neq \mathbf{C}^{n}$, the simplest choice of $q$ is just $q=-\log d_{\Omega}$. Then $q>-\infty$ everywhere. However, if $\Omega=\mathbf{C}^{n}$, then it is usually not convenient to use $q=-\log d_{\Omega}=-\infty$, because with this choice of $q$, the behavior of $f$ at infinity would influence the local properties of the functions we construct. In this case it is best just to take $q=0$.

The functions $u_{f, q}$ and $U_{f, q}$ are well defined and $<+\infty$ in $\Omega_{q}$, thanks to our assumption $\exp (-q(x)) \leqslant d_{\Omega}(x)$. We define them to be $+\infty$ outside $\Omega_{q}$.

Clearly $u_{f} \leqslant U_{f}$, and we shall see that there are inequalities in the opposite direction. We can note quickly that $u_{a f+b g}=a u_{f}+b u_{g}$ for non-negative $a, b$, even for real $a, b$, which implies that the function $u_{f}$ depends linearly of $f$ in the linear space of all Borel measurable functions which are integrable on spheres, thus in particular on the space $\delta P S H(\Omega)$ of delta-plurisubharmonic functions, i.e., the vector space spanned by those plurisubharmonic functions which are not identically minus infinity in any open component of $\Omega$ (see the end of section 1.2). We shall see that this implies that the Lelong number is a linear function on $\delta P S H(\Omega)$. As 
to the function $U_{f}$ we can only say that $U_{a f+b g} \leqslant a U_{f}+b U_{g}$ for $a, b \geqslant 0$, which implies that $U_{f}$ is a convex function of $f$, and the Lelong number a concave function of $f$. But when it comes to the maximum of two functions, we have $U_{\max (f, g)}=\max \left(U_{f}, U_{g}\right)$ which implies that $\nu_{\max (f, g)}=\min \left(\nu_{f}, \nu_{g}\right)$, whereas for the mean we can say only that $u_{\max (f, g)} \geqslant \max \left(u_{f}, u_{g}\right)$ which implies that $\nu_{\max (f, g)} \leqslant \min \left(\nu_{f}, \nu_{g}\right)$. It is therefore useful to know that the Lelong number can be defined by either $u_{f}$ or $U_{f}$, because this enables us to use the best properties of either one.

We can define the Lelong number as the slope at minus infinity of the function $t \mapsto$ $u(x, t)$. As a consequence of the maximum principle, $u(x, t)$ and $U(x, t)$ are increasing in $t$; by Hadamard's three-circle theorem, they are convex functions of $t$. Therefore their slopes at $-\infty$ exist:

$$
\nu_{f}(x)=\lim _{t \rightarrow-\infty} \frac{u(x, t)}{t} \text { and } \quad N_{f}(x)=\lim _{t \rightarrow-\infty} \frac{U(x, t)}{t}
$$

both exist. This follows from the fact that the slopes

$$
\frac{u(x, t)-u\left(x, t_{0}\right)}{t-t_{0}} \text { and } \frac{U(x, t)-U\left(x, t_{0}\right)}{t-t_{0}}
$$

are increasing in $t$. The first limit $\nu_{f}(x)$ is the Lelong number of $f$ at $x$, and the definition we shall use in this chapter. The definition (2.1.1) of the Lelong number as the density of a measure is equivalent to (2.2.4) as can be proved without difficulty using Stokes' theorem (Kiselman [1979]). To see this we shall calculate the mean density assuming that $f$ is of class $C^{2}$. We first express the mass of $\mu$ in a ball in terms of the derivative of $u$ :

$$
\mu(x+r B)=\frac{1}{2 \pi} \int_{x+r B} \Delta f=\frac{1}{2 \pi} \int_{x+r S} \frac{\partial f}{\partial r} d S=\frac{1}{2 \pi} \frac{\partial u}{\partial t} \frac{d t}{d r} \int_{r S} d S=\frac{1}{2 \pi r} \frac{\partial u}{\partial t} \int_{r S} d S .
$$

We now compare with the integral over a ball of lower dimension:

$$
\int_{r S} d S=r^{2 n-1} \int_{S} d S=2 \pi r^{2 n-1} \int_{B^{2 n-2}} d \lambda_{2 n-2}=2 \pi r \int_{r B^{2 n-2}} d \lambda_{2 n-2}=2 \pi r \lambda_{2 n-2}\left(r B^{2 n-2}\right) .
$$

Note that we use the unit sphere of dimension $2 n-1$ and the unit ball of dimension $2 n-2$ here; the remarkable fact is that the quotient

$$
\frac{\operatorname{area}\left(S^{2 n-1}\right)}{\operatorname{volume}\left(B^{2 n-2}\right)}=2 \pi
$$

is independent of the dimension. The mean density $\mu(x+r B) / \lambda_{2 n-2}\left(r B \cap \mathbf{C}^{n-1}\right)$ is therefore equal to the slope $\partial u / \partial t$ at the point $t=\log r$, and the density at the point $x$ is equal to the limit $\lim _{t \rightarrow-\infty} \partial u / \partial t(x, t)$. We can now get rid of the extra assumption that $f$ is of class $C^{2}$, the derivative of $u$ being replaced by the derivative from the right (we use closed balls).

Since $u_{f} \leqslant U_{f}$ we have $\nu_{f}(x) \geqslant N_{f}(x)$. We shall now see that the two numbers are equal. To this end we shall use Harnack's inequality, which takes the form

$$
\frac{1+|x| / r}{(1-|x| / r)^{m-1}} h(0) \leqslant h(x) \leqslant \frac{1-|x| / r}{(1+|x| / r)^{m-1}} h(0)
$$


for harmonic functions which satisfy $h \leqslant 0$ in the ball of radius $r$ in $\mathbf{R}^{m}$. If $f$ is subharmonic in a neighborhood of the closed ball $e^{s} B$ in $\mathbf{C}^{n}$, we can consider its harmonic majorant $h$ there, which satisfies $f(x) \leqslant h(x)$ and

$$
h(0)=f_{z \in S} h\left(e^{s} z\right)=f_{z \in S} f\left(e^{s} z\right)=u(0, s) .
$$

Therefore

$$
U(0, t)=\sup _{e^{t} S} f \leqslant \sup _{e^{t} S} h \leqslant \frac{1-e^{t-s}}{\left(1+e^{t-s}\right)^{2 n-1}} u(0, s), \quad t<s,
$$

provided only $f \leqslant 0$ in $e^{s} B$. If we apply this inequality to the function $f-U(0, s)$, which is non-positive in $e^{s} B$ by definition, we get, writing $U(t)$ instead of $U(0, t)$ for simplicity:

$$
U(t)-U(s) \leqslant \frac{1-e^{t-s}}{\left(1+e^{t-s}\right)^{2 n-1}}(u(s)-U(s))
$$

equivalently,

$$
U(t) \leqslant\left(1-\lambda_{t-s}\right) U(s)+\lambda_{t-s} u(s), \quad t<s,
$$

where $\lambda_{t}$ is defined for $t<0$ as

$$
\lambda_{t}=\frac{1-e^{t}}{\left(1+e^{t}\right)^{2 n-1}} .
$$

We can now prove that the two limits in (2.2.4) are equal. As already noted, $\nu_{f}(x) \geqslant$ $N_{f}(x)$. In the other direction we can take for instance $s=t+1$ in (2.2.6) to obtain the estimate

$$
U(t) \leqslant\left(1-\lambda_{-1}\right) U(t+1)+\lambda_{-1} u(t+1),
$$

whence

$$
\frac{U(t)}{t} \geqslant\left(1-\lambda_{-1}\right) \frac{U(t+1)}{t}+\lambda_{-1} \frac{u(t+1)}{t}, \quad t<0 .
$$

Letting $t$ tend to $-\infty$ we see that $N_{f}(x) \geqslant \nu_{f}(x)$.

To any given $f, q \in P S H(\Omega)$ we define

$$
\varphi_{\tau}(x)=\inf _{t}\left[u_{f}(x, t)-\tau \operatorname{Re} t\right], \quad x \in \Omega, \tau \geqslant 0 .
$$

In view of our convention that $u_{f}(x, t)=+\infty$ if $(x, t) \notin \Omega_{q}$, the infimum is effectively only over those $t$ that satisfy $\operatorname{Re} t<-q(x)$. The function $\tau \mapsto-\varphi_{\tau}(x)$ is the Fenchel transform of $\mathbf{R} \ni t \mapsto u_{f}(x, t)$; cf. (3.4.1). We assume all the time that $e^{-q(x)}$ does not exceed the distance $d_{\Omega}(x)$ from $x$ to the boundary of $\Omega$, so that $u_{f}$ is well defined. The function $(x, t) \mapsto u_{f}(x, t)-\tau \operatorname{Re} t$ is plurisubharmonic in $\Omega_{q}$ and independent of the imaginary part of $t$. Therefore the minimum principle, Theorem 1.3.7, can be applied and yields that $\varphi_{\tau}$ is plurisubharmonic in $\Omega$.

Example. Let us look at the simplest example: $f(x)=\log |x|, x \in \mathbf{C}^{n}$. We choose $q=0$ and form $U_{f}(x, t)=\log \left(e^{t}+|x|\right)$ for $t<q(x)=0$. Then $\varphi_{\tau}(x)=\inf _{t<0}\left(U_{f}(x, t)-\tau t\right)$ can be calculated explicitly: it is $\varphi_{\tau}(x)=(1-\tau) \log |x|+C_{\tau}$ for $0 \leqslant \tau<1$, where $C_{\tau}$ is a constant which depends on the parameter $\tau$, and $\varphi_{\tau}(x)=\log (1+|x|)$ for $\tau \geqslant 1$. Thus the Lelong number of $\varphi_{\tau}$ at the origin is $\max (1-\tau, 0)$ for all $\tau \geqslant 0$.

There is no apparent reason why the Lelong number of the plurisubharmonic function $\varphi_{\tau}$ at $x$ should be a function of $\nu_{f}(x)$ and $\tau$; it could as well depend in some other way on the behavior of $f$ near $x$. However, it turns out that the simple formula for the Lelong number of $\varphi_{\tau}$ in the example holds quite generally: 
Theorem 2.2.1 (Kiselman [1979]). Let $f, q \in P S H(\Omega)$ with $q \geqslant-\log d_{\Omega}$. Define $\varphi_{\tau}$ by (2.2.2) and (2.2.7). Then $\varphi_{\tau} \in P S H(\Omega)$. If $\nu_{q}(x)=0$, then the Lelong number of $\varphi_{\tau}$ is

$$
\nu_{\varphi_{\tau}}(x)=\max \left(\nu_{f}(x)-\tau, 0\right)=\left(\nu_{f}(x)-\tau\right)^{+}, \quad x \in \Omega, \tau \geqslant 0 .
$$

We can also use the function $U_{f}$ instead of $u_{f}$ in the construction; the proof is the same. If $\tau<0$, then of course $\nu_{\varphi_{\tau}}(x)=+\infty$.

We shall give a simplified proof of Theorem 2.2.1 under the slightly stronger hypothesis that $q(x)>-\infty$. This is quite enough for the applications we have in mind. (As soon as $\Omega \neq \mathbf{C}^{n}$, we must indeed have $q>-\infty$ everywhere.)

Lemma 2.2.2. With $f$ and $\varphi_{\tau}$ as in Theorem 2.2.1 we have

$$
\nu_{\varphi_{\tau}}(x) \geqslant \nu_{f}(x)-\tau \text {. }
$$

Proof. We first note that by the definition of $\varphi_{\tau}$, we have for any $t^{\prime}$

$$
\varphi_{\tau}(y) \leqslant u_{f}\left(y, t^{\prime}\right)-t^{\prime} \tau \text {. }
$$

Taking the mean over the sphere $x+e^{t} S$ then gives

$$
\begin{aligned}
u_{\varphi_{\tau}}(x, t) & =f_{z \in S} \varphi_{\tau}\left(x+z e^{t}\right) \leqslant f_{z \in S} f_{w \in S} f\left(x+z e^{t}+w e^{t^{\prime}}\right)-t^{\prime} \tau \\
& \leqslant f_{z \in S} f\left(x+z e^{t^{\prime \prime}}\right)-t^{\prime} \tau=u_{f}\left(x, t^{\prime \prime}\right)-t^{\prime} \tau .
\end{aligned}
$$

Here $t$ and $t^{\prime}$ are arbitrary and $t^{\prime \prime}$ is determined from them by the equation $e^{t^{\prime \prime}}=e^{t}+e^{t^{\prime}}$. The only interesting choice is $t=t^{\prime}$, so that $t^{\prime \prime}=t+\log 2$. Thus

$$
\frac{u_{\varphi_{\tau}}(x, t)}{t} \geqslant \frac{u_{f}(x, t+\log 2)}{t}-\tau, \quad t<0
$$

and letting $t$ tend to $-\infty$ we get the desired conclusion.

Lemma 2.2.3. With $f$ and $\varphi_{\tau}$ as in Theorem 2.2.1, take a number $\tau>\nu_{f}(x)$. Assume that $q(x)>-\infty$. Then $\varphi_{\tau}(x)>-\infty$. In particular $\nu_{\varphi_{\tau}}(x)=0$.

Proof. Since $\nu_{f}(x)<\tau<+\infty, f$ is not equal to $-\infty$ identically in a neighborhood of $x$. The value $\varphi_{\tau}(x)$ is the infimum of the convex function $u_{f}(x, t)-t \tau$ of the real variable $t$ when $t$ varies in the interval $]-\infty,-q(x)[$. This interval is by hypothesis bounded from the right. Moreover by the choice of $\tau$, the function is strictly decreasing when $t \ll 0$. Thus its infimum is finite.

Proof of Theorem 2.2.1, assuming that $q(x)$ is finite. The proof consists of the following steps (cf. Kiselman [1992]). First we note that $\varphi_{\tau}$ is a concave function of $\tau$ with $\varphi_{0}=f$. Therefore $\nu_{\varphi_{\tau}}(x)$ is a convex function of $\tau$ taking the value $\nu_{f}(x)$ for $\tau=0$, for the Lelong 
number is as we have seen a linear function of $f$, the limit of $u(x, t) / t$. Second we see from Lemma 2.2.2 that $\nu_{\varphi_{\tau}}(x) \geqslant \nu_{f}(x)-\tau$. Third we know from Lemma 2.2.3 (if $\nu_{f}(x)$ is finite) that $\nu_{\varphi_{\tau}}(x)=0$ if $\tau>\nu_{f}(x)$. Now the only convex function of $\tau$ which has these properties is $\tau \mapsto\left(\nu_{f}(x)-\tau\right)^{+}$.

\subsection{The Hörmander-Bombieri theorem and the integrability index}

The purpose of this chapter is to show how the singularities of plurisubharmonic functions give rise to, and can be described by, analytic varieties. To do so we shall of course need a method to construct varieties defined by a given plurisubharmonic function. This method is the technique of solving the $\bar{\partial}$ equation using plurisubharmonic functions as weights, most elegantly expressed by the Hörmander-Bombieri theorem:

Theorem 2.3.1. Let $\Omega$ be a pseudoconvex open set in $\mathbf{C}^{n}$, and let $\varphi \in P S H(\Omega)$. For every $a \in \Omega$ such that $e^{-\varphi} \in L_{\text {loc }}^{2}(a)$ there exists a holomorphic function $h \in \mathcal{O}(\Omega)$ such that $h(a)=1$ and

$$
\int_{\Omega}|h|^{2} e^{-2 \varphi}\left(1+|z|^{2}\right)^{-3 n} d \lambda_{2 n}(z)<+\infty
$$

Here $L_{l o c}^{2}(a)$ denotes the set of all functions that are square integrable in some neighborhood of the point $a$.

For the proof see Hörmander [1990: Theorem 4.4.4]. (The exponent $-3 n$ can be improved to $-n-\varepsilon$ for any positive $\varepsilon$; see Hörmander [forthc.]. This is, however, not important in a local study like ours.) Let us denote by $\mathcal{O}(\Omega, \varphi)$ the set of all holomorphic functions $h$ in $\Omega$ which satisfy condition (2.3.1) for a given function $\varphi$. The intersection

$$
V(\varphi)=\bigcap_{h}\left(h^{-1}(0) ; h \in \mathcal{O}(\Omega, \varphi)\right)
$$

is an intersection of zero sets of holomorphic functions, and therefore itself an analytic set. Let us define

$$
I(\varphi)=\left\{a \in \Omega ; e^{-\varphi} \notin L_{l o c}^{2}(a)\right\} .
$$

With this notation the theorem says that $V(\varphi) \subset I(\varphi)$. It is however obvious that $I(\varphi) \subset$ $V(\varphi)$.

In view of this theorem it is natural to measure the singularity of a plurisubharmonic function $\varphi$ at a point $a$ by its integrability index $\iota_{\varphi}(a)$ :

$$
\iota_{\varphi}(a)=\inf _{t>0}\left[t ; e^{-\varphi / t} \in L_{l o c}^{2}(a)\right] .
$$

It is easy to see that if $e^{-\varphi / t} \in L_{l o c}^{2}(a)$, then also $e^{-\varphi / s} \in L_{l o c}^{2}(a)$ for every $s>t$. Thus the set of $t>0$ such that $e^{-\varphi / t} \in L_{l o c}^{2}(a)$ is an interval, either $\left[\iota_{\varphi}(a),+\infty[\right.$ or $] \iota_{\varphi}(a),+\infty[$. In all examples I have seen, it is an open interval. It seems to be unknown whether it is always open for plurisubharmonic $\varphi$.

Let $\Phi$ be an arbitrary subset of $P S H(\Omega)$ and $\kappa$ a functional on $\Phi$ in the sense that there is given a function $\kappa_{\varphi}: \Omega \rightarrow[0,+\infty]$ for every $\varphi \in \Phi$. We introduce a notation for the superlevel sets of such functionals:

$$
E_{c}^{\kappa}(\varphi)=\left\{a \in \Omega ; \kappa_{\varphi}(a) \geqslant c\right\}, \quad c \geqslant 0 .
$$


The superlevel sets of the integrability index are analytic varieties. In fact, by the definition of $\iota$

$$
E_{c}^{\iota}(\varphi) \subset I(\varphi / t) \subset E_{t}^{\iota}(\varphi), \quad 0<t<c .
$$

Using the Hörmander-Bombieri theorem we see that

$$
E_{c}^{\iota}(\varphi) \subset V(\varphi / t) \subset E_{t}^{\iota}(\varphi), \quad 0<t<c .
$$

We now note that by the definition of the superlevel set, the intersection of all $E_{t}^{\iota}(\varphi)$ when $t$ varies in the interval $0<t<c$ is just $E_{c}^{\iota}(\varphi)$, so that

$$
E_{c}^{\iota}(\varphi)=\bigcap_{0<t<c} V(\varphi / t), \quad c \geqslant 0
$$

Suppose that $\kappa$ is a functional which is comparable to the integrability index in the sense that the inequality

$$
s \iota_{\varphi}(x) \leqslant \kappa_{\varphi}(x) \leqslant \iota_{\varphi}(x), \quad \varphi \in \Phi, x \in \Omega,
$$

holds for some positive constants $s$ and $t$. Then there is of course a relation between the superlevel sets of the two functionals:

$$
E_{t c}^{\kappa}(\varphi) \subset E_{c}^{\iota}(\varphi) \subset E_{s c}^{\kappa}(\varphi), \quad \varphi \in \Phi .
$$

If we know that $E_{t c}^{\kappa}(\varphi)=E_{s c}^{\kappa}(\varphi)$, then $E_{t c}^{\kappa}(\varphi)$ equals $E_{c}^{\iota}(\varphi)$ and so is an analytic variety. Of course functions which admit such an interval of constancy in their superlevel sets are very special. But we shall see in the next section that for a set of plurisubharmonic functions such intervals of constancy can appear quite naturally.

We now ask whether the Lelong number is comparable to the integrability index in the sense of (2.3.5). The answer is well-known, but will be quoted here for convenience.

Theorem 2.3.2. If $\varphi \in P S H(\Omega)$ where $\Omega \subset \mathbf{C}^{n}$, and $\nu_{\varphi}(a) \geqslant n$, then $e^{-\varphi} \notin L_{\text {loc }}^{2}(a)$. Thus $E_{n}^{\nu}(\varphi) \subset I(\varphi) \subset V(\varphi)$. In terms of the integrability index we have $\nu_{\varphi}(x) \leqslant n \iota_{\varphi}(x)$.

Proof. This result is contained in Skoda [1972, Proposition 7.1], but it is easy to give a proof using the function $U=U_{\varphi}$ defined by (2.2.3). If $\nu_{\varphi}(a) \geqslant n$, then the slope of $t \mapsto U(a, t)$ at minus infinity is at least $n$, and we have

$$
U(a, t) \leqslant U\left(a, t_{0}\right)+n\left(t-t_{0}\right), \quad t \leqslant t_{0},
$$

for some $t_{0}$. Rewriting this in terms of $\varphi$ we see that

$$
\varphi(z) \leqslant \varphi\left(z_{0}\right)+\log \left(\frac{|z-a|^{n}}{\left|z_{0}-a\right|^{n}}\right), \quad|z-a| \leqslant\left|z_{0}-a\right|,
$$

for a suitable point $z_{0}$ on the sphere $|z-a|=e^{t_{0}}$, or equivalently

$$
e^{-2 \varphi(z)} \geqslant e^{-2 \varphi\left(z_{0}\right)} \frac{\left|z_{0}-a\right|^{2 n}}{|z-a|^{2 n}}
$$

where the right-hand side is a non-integrable function near $a$.

In the other direction we have: 
Theorem 2.3.3. If $\varphi \in P S H(\Omega)$ has a finite value at a point $a \in \Omega$, then $e^{-\varphi} \in L_{\text {loc }}^{2}(a)$.

For the proof of this result, see Hörmander [1990: Theorem 4.4.5]. The theorem says that $I(\varphi)$ is contained in the polar set $P(\varphi)=\varphi^{-1}(-\infty)$ of $\varphi$, thus $I(\varphi) \subset P(\varphi)$.

Combining Theorems 2.3.1, 2.3.2 and 2.3.3 we see that

$$
E_{n c}^{\nu}(\varphi) \subset V(\varphi / c) \subset P(\varphi), \quad c>0
$$

A stronger result in the same direction is

Theorem 2.3.4. If $\nu_{\varphi}(a)<1$, then $e^{-\varphi} \in L_{\text {loc }}^{2}(a)$. Thus $I(\varphi) \subset E_{1}^{\nu}(\varphi)$. Also $\iota_{\varphi}(x) \leqslant \nu_{\varphi}(x)$.

For the proof see Skoda [1972: Proposition 7.1].

Combining Theorems 2.3.1, 2.3.2 and 2.3.4 we get

Theorem 2.3.5. The Lelong number $\nu$ is comparable to the integrability index $\iota$ in the sense of (2.3.5), more precisely,

$$
\iota_{\varphi}(x) \leqslant \nu_{\varphi}(x) \leqslant n \iota_{\varphi}(x), \quad \varphi \in P S H(\Omega), x \in \Omega \subset \mathbf{C}^{n},
$$

and

$$
E_{n c}^{\nu}(\varphi) \subset V(\varphi / c) \subset E_{c}^{\nu}(\varphi) \subset V(n \varphi / c), \quad \varphi \in P S H(\Omega), c>0 .
$$

These inequalities are sharp.

This result is the basis for the analyticity theorems that we shall state. However, the weaker result (2.3.7) is often sufficient.

That the comparison in (2.3.8) between the Lelong number and the integrability index cannot be improved follows from simple examples:

Example 2.3.6. The function

$$
f(z)=\max \left(\log \left|z_{1}\right|^{a}, \log \left|z_{2}\right|^{b}\right), \quad z \in \mathbf{C}^{2},
$$

has integrability index $\iota_{f}(0)=a b /(a+b)$ and Lelong number $\nu_{f}(0)=\min (a, b)$. Thus

$$
\left.\left.\frac{\nu_{f}(0)}{\iota_{f}(0)}=\frac{a+b}{\max (a, b)}=\frac{a^{-1}+b^{-1}}{\max \left(a^{-1}, b^{-1}\right)} \in\right] 1,2\right] .
$$

A little more generally, if we take $1 \leqslant k \leqslant n$ and positive numbers $a_{1}, \ldots, a_{k}$ and define

$$
f(z)=\max _{1 \leqslant j \leqslant k} \log \left|z_{j}\right|^{a_{j}}, \quad z \in \mathbf{C}^{n},
$$

then $\iota_{f}(0)=\left(\sum a_{j}^{-1}\right)^{-1}$ and $\nu_{f}(0)=\min a_{j}$, so that

$$
\frac{\nu_{f}(0)}{\iota_{f}(0)}=\frac{\sum a_{j}^{-1}}{\max a_{j}^{-1}} \in[1, n] .
$$


(These formulas hold if we define $a_{j}^{-1}=0$ for $j=k+1, \ldots, n$.) Clearly the quotient $\nu_{f} / \iota_{f}$ can assume all values in the closed interval $[1, n]$ (we allow $k=1$ and $k=n$ ). Thus (2.3.8) is sharp.

\subsection{Analyticity theorems for sets of plurisubharmonic functions}

Let $\Omega$ be an open set in $\mathbf{C}^{n}$ and $\Phi$ an arbitrary subset of $P S H(\Omega)$. Let $\kappa$ be a functional on $\Phi$ which is comparable to the integrability index in the sense that (2.3.5) holds for some positive constants $s$ and $t$. Then we get from (2.3.6) and (2.3.7):

$$
\bigcap_{\varphi \in \Phi} E_{t c}^{\kappa}(\varphi) \subset \bigcap_{\varphi \in \Phi} E_{c}^{\iota}(\varphi) \subset \bigcap_{\varphi \in \Phi} E_{s c}^{\kappa}(\varphi) \subset \bigcap_{\varphi \in \Phi} P(\varphi) .
$$

It is convenient to introduce a notation for these sets:

$$
E_{c}^{\kappa}(\Phi)=\bigcap_{\varphi \in \Phi} E_{c}^{\kappa}(\varphi)
$$

for any functional $\kappa$. If we define the value of the functional on the whole set $\Phi$ as

$$
\kappa_{\Phi}(x)=\inf _{\varphi \in \Phi} \kappa_{\varphi}(x)
$$

then $E_{c}^{\kappa}(\Phi)$ is just the superlevel set of $\kappa_{\Phi}$. We can also define the polar set of $\Phi$ as

$$
P(\Phi)=\bigcap_{\varphi \in \Phi} P(\varphi)
$$

With this notation we can write (2.4.1) as

$$
E_{t c}^{\kappa}(\Phi) \subset E_{c}^{\iota}(\Phi) \subset E_{s c}^{\kappa}(\Phi) \subset P(\Phi)
$$

Theorem 2.4.1. Let $\kappa$ be a functional on a subset $\Phi$ of $P S H(\Omega)$ which is comparable to the integrability index in the sense that (2.3.5) holds for some positive constants $s$ and $t$. If the superlevel sets $E_{c}^{\kappa}(\Phi)$ are independent of c over an interval of sufficiently large logarithmic length, viz. if $E_{s c}^{\kappa}(\Phi)=E_{t c}^{\kappa}(\Phi)$, then $E_{s c}^{\kappa}(\Phi)$ is an analytic variety. A little more generally, if $Y$ is an analytic subset of $\Omega$ and $Y \cap E_{s c}^{\kappa}(\Phi)=Y \cap E_{t c}^{\kappa}(\Phi)$, then $Y \cap E_{s c}^{\kappa}(\Phi)$ is analytic.

Proof. Since the result is local, we can assume $\Omega$ to be pseudoconvex. The inclusions (2.4.2) then show that $E_{s c}^{\kappa}(\Phi)=E_{c}^{\iota}(\Phi)$, which is the set of common zeros of a family of holomorphic functions in $\Omega$; see (2.3.4). Similarly, $Y \cap E_{s c}^{\kappa}(\Phi)=Y \cap E_{c}^{\iota}(\Phi)$.

Theorem 2.4.2. Let $\Omega, \kappa$ and $\Phi$ be as in Theorem 2.4.1, and assume in addition that $\kappa$ is positively homogeneous, i.e., $\varphi \in \Phi$ and $t>0$ implies $t \varphi \in \Phi$ and $\kappa_{t \varphi}(a)=t \kappa_{\varphi}(a)$. Let $Y$ be an analytic subset of $\Omega$, and let $X$ be a subset of $Y$. Assume that

$$
\inf _{x \in X} \kappa_{\varphi}(x)>0 \quad \text { for all } \varphi \in \Phi
$$

and

$$
\text { for every } x \in Y \backslash X \text { there exists a function } \varphi \in \Phi \text { such that } \kappa_{\varphi}(x)=0 \text {. }
$$


Then $X$ is an analytic set.

Proof. Define $\psi=\varphi / \varepsilon_{\varphi}$, where $\varepsilon_{\varphi}=\inf _{x \in X} \kappa_{\varphi}(x)>0$, and let $\Psi$ be the set of all functions $\psi$ obtained in this way. Then $\kappa_{\psi}(x) \geqslant 1$ for every $\psi \in \Psi$ and every $x \in X$. Using the notation for superlevel sets we can write this as

$$
X \subset E_{1}^{\kappa}(\Psi) \subset E_{c}^{\kappa}(\Psi)
$$

for all $c$ with $0 \leqslant c \leqslant 1$.

On the other hand, if $x \in Y \backslash X$, then by (2.4.4) there is a $\varphi \in \Phi$ such that $\kappa_{\varphi}(x)=0$. Thus $\psi=\varphi / \varepsilon_{\varphi}$ is in $\Psi$ and $\kappa_{\psi}(x)=\kappa_{\varphi}(x) / \varepsilon_{\varphi}=0$. So $x \notin E_{c}^{\kappa}(\psi), c$ being any positive number. Thus

$$
x \notin E_{c}^{\kappa}(\Psi)=\bigcap_{\psi \in \Psi} E_{c}^{\kappa}(\psi) .
$$

Therefore $E_{c}^{\kappa}(\Psi) \cap Y \subset X$ for every $c>0$. Combining this with the first part of the proof we see that $E_{c}^{\kappa}(\Psi) \cap Y=X$ for all $c$ satisfying $0<c \leqslant 1$. Hence $E_{c}^{\kappa}(\Psi) \cap Y$ is constant for these $c$ and Theorem 2.4.1 yields that $X$ is analytic.

A particular case of Theorem 2.4.2 is when we can associate with a given function or current a family of plurisubharmonic functions on which our functional takes values that we can control. The following result is of this character. It holds also for functionals which have only a loose connection to the integrability index or the Lelong number; more precisely functionals which are zero at the same time as the integrability index in a semiuniform way:

Theorem 2.4.3. Let $\Phi=\left\{\varphi_{\alpha} ; \alpha \in A\right\}$ be a set of plurisubharmonic functions in an open set $\Omega$, and let $\kappa$ be a functional on $\Phi$ which is weakly comparable to the integrability index in the sense that

$$
\text { for every } \varepsilon>0 \text { there is a } \delta>0 \text { such that } \varphi \in \Phi, \iota_{\varphi}(x)<\delta \text { implies } \kappa_{\varphi}(x)<\varepsilon \text {, }
$$

and

$$
\varphi \in \Phi, \kappa_{\varphi}(x)=0 \text { implies } \iota_{\varphi}(x)=0 .
$$

Suppose that the values $\kappa_{\varphi_{\alpha}}(x)$ are given by a formula $\kappa_{\varphi_{\alpha}}(x)=G(H(x), \alpha)$ for some functions $G:[0,+\infty] \times A \rightarrow[0,+\infty]$ and $H: \Omega \rightarrow[0,+\infty]$. We assume that $c \mapsto G(c, \alpha)$ is increasing, and that there exists a number $c_{0}$ such that $G\left(c_{0}, \alpha\right)>0$ for all $\alpha \in A$. Finally we suppose that for every $c<c_{0}$ there is an $\alpha \in A$ such that $G(c, \alpha)=0$. Then the superlevel set $\left\{x ; H(x) \geqslant c_{0}\right\}$ is analytic.

Proof. We shall apply Theorem 2.4.2 to $X=\left\{x ; H(x) \geqslant c_{0}\right\}$. First we note that

$$
\inf _{x \in X} \kappa_{\varphi_{\alpha}}(x)=\inf _{x \in X} G(H(x), \alpha) \geqslant G\left(c_{0}, \alpha\right)=\varepsilon_{\alpha}>0
$$

for any $\alpha \in A$. Hence $\iota_{\varphi_{\alpha}}(x) \geqslant \delta_{\alpha}>0$, which means that (2.4.3) holds for $\iota$. Next, if $x \notin X$, then $c=H(x)<c_{0}$ and there is an $\alpha$ such that $G(c, \alpha)=0$. We get

$$
\kappa_{\varphi_{\alpha}}(x)=G(H(x), \alpha)=G(c, \alpha)=0
$$


hence also $\iota_{\varphi_{\alpha}}(x)=0$, so that (2.4.4) holds for $\iota$. Thus Theorem 2.4.2, applied to $\iota$, shows that $X$ is analytic.

This theorem contains the classical theorem of Siu [1974]. For if we let $\kappa_{\varphi}(x)=\nu_{\varphi}(x)$, $A=\left[0, c_{0}\left[, G(c, \alpha)=(c-\alpha)^{+}, H(x)=\nu_{f}(x)\right.\right.$, and define $\varphi_{\alpha}$ as

$$
\varphi_{\alpha}(x)=\inf _{t}\left[u_{f}(x, t)-t \alpha ;(x, t) \in \Omega_{q}\right], \quad x \in \Omega, \alpha \in\left[0, c_{0}[,\right.
$$

then by Theorem 2.2.1,

$$
\nu_{\varphi_{\alpha}}(x)=\left(\nu_{f}(x)-\alpha\right)^{+}=G(H(x), \alpha)
$$

The function $G(c, \alpha)=(c-\alpha)^{+}$satisfies the hypotheses of Theorem 2.4.3, so it follows that the superlevel set $\left\{x ; \nu_{f}(x) \geqslant c_{0}\right\}$ is an analytic variety. The singularities of the $\varphi_{\alpha}$ are the same as those of $f$, but attenuated to some degree as shown by the formula. This attenuation is the reason behind their usefulness in proving Siu's theorem. 


\section{Chapter 3. Order and type as measures of growth}

\subsection{Introduction}

The notions of order and type of entire functions are classical in complex analysis. They result from a comparison of a given function with standard functions. The purpose of this chapter is to generalize this comparison in such a way that order and type become dual to each other in the sense of convex analysis (section 3.4), and to show that the concept of order so obtained appears as the natural answer to a problem of extrapolation: to extend convex functions from the union of two parallel hyperplanes to as large a set as possible (section 3.7). Then we return to entire functions to consider an analogous extension problem for them (section 3.8).

It is shown that the relative order of one function with respect to another can always be calculated from the growth of its Taylor coefficients (section 3.6). This is true for the type only if the growth is sufficiently regular (see Kiselman [1993]).

In Kiselman [1983] I studied order and type from this point of view, using methods from my paper [1981]. For earlier developments see the references in that paper. See also Kiselman [1984, 1986]. A different approach to the relation between maximum modulus and Taylor coefficients is presented in Freund and Görlich [1985]. Halvarsson [forthc.] has proved an extension theorem for entire functions with estimates both from above and from below. He has also studied the dependence of the order on parameters.

\subsection{Order and type in classical complex analysis}

Let $h$ be an entire function in $\mathbf{C}^{n}, h \in \mathcal{O}\left(\mathbf{C}^{n}\right)$. Its order and type are defined classically by comparing $h$ with the function $\exp \left(b|z|^{a}\right)$ for various choices of the parameters $a$ and $b$. More precisely, one considers first estimates

$$
|h(z)| \leqslant C_{a} e^{|z|^{a}}, \quad z \in \mathbf{C}^{n}
$$

and defines the order $\rho$ as the infimum of all numbers $a$ for which such an estimate holds $(0<a<+\infty ; 0 \leqslant \rho \leqslant+\infty)$. In the case where $0<\rho<+\infty$ one then considers all numbers $b$ such that

$$
|h(z)| \leqslant C_{b} e^{b|z|^{\rho}}, \quad z \in \mathbf{C}^{n},
$$

for some constant $C_{b}$. The type (with respect to the order $\rho$ ) is then the infimum $\sigma$ of all such numbers $b(0<b<+\infty ; 0 \leqslant \sigma \leqslant+\infty)$.

For the order we have the formula

$$
\rho=\operatorname{order}(h)=\limsup _{r \rightarrow+\infty} \sup _{|z|=r} \frac{\log \log |h(z)|}{\log r} .
$$


Now $\sup _{|z|=r} \log |h(z)|$ is a convex function of $\log r$ in view of the Hadamard three-circle theorem, so it is natural to consider the function

$$
f(t)=\sup _{|z|=e^{t}} \log |h(z)|, \quad t \in \mathbf{R}
$$

we shall call it the growth function of $h$. The definition of order then means that we consider all numbers $a$ such that

$$
f(t) \leqslant e^{a t}+C_{a}, \quad t \in \mathbf{R},
$$

for some constant $C_{a}$, and then define the order as the infimum of all such numbers $a$. (The role of the constant $C_{a}$ is to eliminate all influence of values of $f$ at any particular point.) Similarly, the type (for order $\rho$ ) is the infimum of all numbers $b$ such that

$$
f(t) \leqslant b e^{\rho t}+C_{b}, \quad t \in \mathbf{R} .
$$

Now this leads naturally to the idea of comparing with some other function $g$ instead of the exponential function $g(t)=e^{t}$. So we might want to consider all numbers $a$ such that

$$
f(t) \leqslant g(a t)+C_{a}, \quad t \in \mathbf{R},
$$

and then take the infimum of all $a$.

For reasons which will become clear when we come to the duality between order and type, it is desirable to change this inequality to

$$
f(t) \leqslant \frac{1}{a} g(a t)+C_{a}, \quad t \in \mathbf{R} .
$$

Now in the classical case, when $g(t)=e^{t}$, the factor $1 / a$ does not make any difference whatsoever, for in this case we see that for any $a>0$ and any $b>a$ there is a constant $C_{a, b}$ such that

$$
g(a t) \leqslant \frac{1}{b} g(b t)+C_{a, b} \quad \text { and } \quad \frac{1}{a} g(a t) \leqslant g(b t)+C_{a, b} .
$$

This implies that comparisons with $g(a t)$ and with $g(a t) / a$ give identical infima. But of course there exist functions $g$ such that this is not true (e.g., $g(t)=t)$, and then (3.2.2) and (3.2.3) lead to different definitions of the order.

\subsection{Relative order and type of convex functions}

Definition 3.3.1. Let $f, g: E \rightarrow[-\infty,+\infty]$ be two functions defined on a real vector space $E$. We consider inequalities of the form

$$
f(x) \leqslant \frac{1}{a} g(a x)+c, \quad x \in E,
$$

where $a$ is a positive constant and $c$ a real constant. We shall call the infimum of all positive numbers $a$ such that (3.3.1) holds for some constant $c$ the order of $f$ relative to $g$, and denote it by $\rho=\operatorname{order}(f: g)$. 
Examples. The motivating example is

$$
\operatorname{order}\left(t \mapsto e^{A t}: t \mapsto e^{t}\right)=A
$$

for all positive numbers $A$. Trivial examples are: $\operatorname{order}(a: b)=0$ if $a$ and $b$ are finite constants; $\operatorname{order}(f:+\infty)=0$; $\operatorname{order}(-\infty: g)=0 ; \operatorname{order}(f:-\infty)=+\infty$ except if $f$ is identically $-\infty$; order $(+\infty: g)=+\infty$ except if $g$ is identically $+\infty$.

If $g$ is convex, we know that

$$
\frac{1}{a} g(a x) \leqslant \frac{1-t}{a} g(0)+\frac{t}{a} g(b x)=\left(\frac{1}{a}-\frac{1}{b}\right) g(0)+\frac{1}{b} g(b x), \quad x \in E,
$$

if $0<a<b$ and $a x=(1-t) \cdot 0+t b x$, i.e., $t=a / b$. Here the sign $\dot{+}$ denotes upper addition, which is an extension of the usual addition from $\mathbf{R}^{2}$ to $[-\infty,+\infty]^{2}$; it satisfies $(+\infty) \dot{+}(-\infty)=+\infty$. Similarly we define lower addition as the extension of + which satisfies $(+\infty)+(-\infty)=-\infty$. If $g(0)=+\infty$, the inequality (3.3.2) is without interest, but if $g(0)<+\infty$, it shows that the inequality (3.3.1) for a particular $a$ implies the same inequality with $a$ replaced by $b$ for any $b>a$. The set of all numbers $a, 0<a<+\infty$, such that (3.3.1) holds is therefore an interval, either $[\rho,+\infty[$ or $] \rho,+\infty$ [ for some $\rho \in[0,+\infty]$.

So although Definition 3.3.1 has a sense for all $f$ and $g$, it is often desirable to assume that $g$ is convex with $g(0)<+\infty$ : in this case the order determines the set of all $a$ for which (3.3.1) holds, with the exception of one point, the order itself.

Lemma 3.3.2. Let $f_{y}$ denote the translate of $f$ by the vector $y: f_{y}(x)=f(x-y)$. If one of $f$ and $g$ is convex and real valued, then

$$
\operatorname{order}\left(f_{y}: g\right)=\operatorname{order}\left(f: g_{y}\right)=\operatorname{order}(f: g) \text {. }
$$

In particular order $\left(f_{y}: g_{y}\right)=\operatorname{order}(f: g)$ so that the order is translation invariant and can be defined on affine spaces as soon as one of the functions is convex and real valued.

Proof. If $f$ is convex and real valued, we know that

$$
f(x-y) \leqslant \frac{1}{b} f(b x)+\left(1-\frac{1}{b}\right) f(z)
$$

for any $b>1$, if we choose $z$ such that

$$
x-y=\frac{1}{b} b x+\left(1-\frac{1}{b}\right) z,
$$

i.e., if $z=-y /(1-1 / b)$. If $\operatorname{order}(f: g)=\rho$, there are numbers $a$ arbitrarily close to $\rho$ such that

$$
f(x) \leqslant \frac{1}{a} g(a x)+c .
$$

We then estimate $f$ as follows:

$$
f(x-y) \leqslant \frac{1}{b} f(b x)+\left(1-\frac{1}{b}\right) f(z) \leqslant \frac{1}{a b} g(a b x)+\frac{1}{b} c+\left(1-\frac{1}{b}\right) f(z) .
$$


Since $f(z)$ is finite and independent of $x$, this shows that $\operatorname{order}\left(f_{y}: g\right) \leqslant a b$, and since $b$ is arbitrarily close to 1 , we see that $\operatorname{order}\left(f_{y}: g\right) \leqslant \rho$. If we apply this result to $f_{y}$, translating by the vector $-y$, we get equality.

Similarly, if $g$ is convex and real valued, we can write

$$
f(x-y) \leqslant \frac{1}{a} g(a(x-y))+c \leqslant \frac{1}{a b} g(a b x)+\frac{1}{a}\left(1-\frac{1}{b}\right) g(z)+c,
$$

where $z=-a y /(1-1 / b)$, thus independent of $x$. This shows that, in this case also, $\operatorname{order}\left(f_{y}: g\right) \leqslant a b$ with $a b$ arbitrarily close to $\rho$.

It remains to consider $\operatorname{order}\left(f: g_{y}\right)$. The arguments are the same as for $\operatorname{order}\left(f_{y}: g\right)$; we omit the proof.

It is easy to give examples of functions with values in $]-\infty,+\infty]$ such that the order is not translation invariant:

Example. Let $f$ be the indicator function of the ball $r B$, i.e., let $f(x)=0$ when $|x| \leqslant r$ and $f(x)=+\infty$ otherwise. Similarly let $g$ be the indicator function of the ball $s B$. In the case where $0<s \leqslant r$ we get

$$
\frac{s}{r} \leqslant \operatorname{order}\left(f_{y}: g_{y}\right)=\frac{s+|y|}{r+|y|} \leqslant 1 .
$$

If $s>r>0$, we have

$$
\operatorname{order}\left(f_{y}: g_{y}\right)= \begin{cases}\frac{s-|y|}{r-|y|} \geqslant \frac{s}{r}>1 & \text { when }|y|<r \\ +\infty & \text { when }|y| \geqslant r\end{cases}
$$

We now consider a generalization of the notion of type in complex analysis.

Definition 3.3.3. Given two functions $f, g: E \rightarrow[-\infty,+\infty]$ on a vector space $E$, we consider inequalities

$$
f(x) \leqslant b g(x)+c, \quad x \in E,
$$

where $b$ is a positive number. We define the type of $f$ relative to $g$ as the infimum of all positive numbers $b$ such that (3.3.3) holds for some constant $c$. We shall denote it by $\sigma=\operatorname{type}(f: g)$.

Example. The motivating example is

$$
\operatorname{type}\left(t \mapsto A e^{\rho t}: t \mapsto e^{\rho t}\right)=A .
$$

The two functions here are the growth functions of the entire functions $\exp \left(A z^{\rho}\right)$ and $\exp \left(z^{\rho}\right)$ if $\rho$ is a natural number, and then $A$ is the classical type with respect to order $\rho$.

If $g$ is bounded from below, the set of all numbers such that (3.3.3) holds is an interval, for as soon as $b_{1}>b$ we have

$$
b g(x)+c=b_{1} g(x)+c-\left(b_{1}-b\right) g(x) \leqslant b_{1} g(x)+c-\left(b_{1}-b\right) \inf g=b_{1} g(x)+c_{1} .
$$


Therefore, although the definition has a sense for all functions, it is clear that it will often be necessary to assume $g$ bounded from below. In this case the type determines all numbers $b$ for which (3.3.3) holds, except the number $\sigma$ itself.

Proximate orders are introduced to give functions of finite order normal type $(0<\sigma<$ $+\infty)$; see Lelong \& Gruman [1986, Appendix II]. The type with respect to a proximate order is a special case of Definition 3.3.3.

A generalization of the classical order and type has been studied, e.g., by Sato [1963] and Juneja, Kapoor \& Bajpai [1976, 1977]. For given integers $p$ and $q$, they study the $(p, q)$-order defined as

$$
\rho_{p q}=\limsup \frac{\log ^{[p]} M(r)}{\log ^{[q]} r}=\limsup \frac{\log ^{[p-1]} f(t)}{\log ^{[q-1]} t},
$$

where $M(r)=\exp f(\log r)$. (Sato considered this only for $q=1$.) Here the brackets indicate iterations of the logarithm function. Now it is easy to see that the $(p, q)$-order is just $\operatorname{order}\left(f_{q}: g_{p}\right)$ where $f_{q}(t)=f\left(\exp ^{[q-1]}(t)\right)$ and $g_{p}(t)=\exp ^{[p-1]}(t)$. Both $f_{q}$ and $g_{p}$ are convex. Their generalization of the notion of type is, however, different from that of Definition 3.3.3. The $(p, q)$-type is

$$
T_{p q}=\limsup \frac{\log ^{[p-1]} M(r)}{\left(\log ^{[q-1]} r\right)^{\rho}}=\limsup \frac{\log ^{[p-2]} f(t)}{\left(\log ^{[q-2]} t\right)^{\rho}} .
$$

For $p \geqslant 3$ this is not the relative type of one convex function with respect to another, but rather an order: it is the order of $f\left(\exp ^{[q-2]} t^{1 / \rho}\right)$ with respect to $\exp ^{[p-2]}(t)$. Therefore our results on order generalize those of the authors mentioned, but our type is different, and some of the earlier results on type can be interpreted as orders in the framework of the present chapter.

\subsection{Order and type in duality}

The notion of order and type as defined in the last section are dual, or conjugate, to each other in the sense of convexity theory. We shall express duality here in terms of the Fenchel transformation: for any function $f: E \rightarrow[-\infty,+\infty]$ we define

$$
\widetilde{f}(\xi)=\sup _{x \in E}(\xi \cdot x-f(x)), \quad \xi \in E^{\prime} .
$$

Here $E$ is a real vector space, and $E^{\prime}$ is any fixed linear subspace of its algebraic dual $E^{*}$. The function $\tilde{f}$ is called the Fenchel transform of $f$; other names are the Legendre transform of $f$, or the conjugate function. It is easy to see that $\tilde{f}$ is convex, lower semicontinuous for the weak-star-topology $\sigma\left(E^{\prime}, E\right)$ and that it never takes the value $-\infty$ except when it is equal to $-\infty$ identically.

Points where $f(x)=+\infty$ do not influence the supremum in (3.4.1). We shall use this fact in the following way. Let $\operatorname{dom} f$ denote the set where $f(x)<+\infty$, the effective domain of $f$. Then for any set $M$ such that $\operatorname{dom} f \subset M \subset E$ we have

$$
\widetilde{f}(\xi)=\sup _{x \in M}(\xi \cdot x-f(x)), \quad \xi \in E^{\prime} .
$$

The inequality

$$
\xi \cdot x \leqslant f(x) \dot{+} \widetilde{f}(\xi),
$$


which follows from (3.4.1), is called Fenchel's inequality (here the sign $\dot{+}$ denotes upper addition; see section 3.3). Applying the transformation twice we get

$$
\widetilde{\widetilde{f}}(x)=\sup _{\xi \in E^{\prime}}(\xi \cdot x-\widetilde{f}(\xi)) \leqslant f(x), \quad x \in E .
$$

Thus always $\widetilde{\widetilde{f}} \leqslant f$; the equality $\widetilde{\widetilde{f}}=f$ holds if and only if $f$ is convex, lower semicontinuous for the weak topology $\sigma\left(E, E^{\prime}\right)$, and takes the value $-\infty$ only if it is $-\infty$ identically. More generally, it follows that $\widetilde{\widetilde{f}}$ is the maximal convex lower semicontinuous minorant of $f$ which never takes the value $-\infty$ except when it is the constant $-\infty$. For these properties of the Fenchel transform see Rockafellar [1970]. Of course $\widetilde{\widetilde{f}}$ depends on the choice of $E^{\prime}$; if $E^{\prime}=\{0\}$, then $\widetilde{\widetilde{f}}$ is the constant inf $f$. If $E=\mathbf{R}^{n}$ it is natural to take $E^{\prime}=E^{*} \cong \mathbf{R}^{n}$; if $E$ is a topological vector space one usually takes $E^{\prime}$ as the topological dual of $E$.

Proposition 3.4.1. Let $f, g: E \rightarrow[-\infty,+\infty]$ be two functions on a vector space $E$. Then

$$
\operatorname{type}(\widetilde{g}: \widetilde{f}) \leqslant \operatorname{order}(f: g) \text {. }
$$

Proof. If order $(f: g)<A$, then $f(x) \leqslant g(a x) / a+c$ for some number $a<A$, and we deduce that $\widetilde{f}(\xi) \geqslant \widetilde{g}(\xi) / a-c$, which we write as $\widetilde{g}(\xi) \leqslant a \widetilde{f}(\xi)+a c$. Therefore type $(\widetilde{g}: \widetilde{f}) \leqslant a<A$.

Proposition 3.4.2. If $f, g: E \rightarrow[-\infty,+\infty]$ are two functions on a vector space $E$, then

$$
\operatorname{order}(\widetilde{g}: \widetilde{f}) \leqslant \operatorname{type}(f: g) \text {. }
$$

Proof. If type $(f: g)<A$ there are numbers $a<A$ and $c$ such that $f(x) \leqslant a g(x)+c$. We take the transformation to obtain $\widetilde{f}(\xi) \geqslant a \widetilde{g}(\xi / a)-c$, which can be written as $\widetilde{g}(\xi) \leqslant \widetilde{f}(a \xi) / a+c / a$. Therefore $\operatorname{order}(\widetilde{g}: \widetilde{f}) \leqslant a<A$.

Theorem 3.4.3. Let $f, g: E \rightarrow[-\infty,+\infty]$ be two functions on a vector space $E$ such that $\widetilde{\widetilde{f}}=f$ and $\widetilde{\widetilde{g}}=g$. Then

$$
\operatorname{order}(\widetilde{g}: \widetilde{f})=\operatorname{type}(f: g) \quad \text { and } \quad \operatorname{type}(\widetilde{g}: \widetilde{f})=\operatorname{order}(f: g) \text {. }
$$

Proof. We just combine Propositions 3.4.1 and 3.4.2.

Corollary 3.4.4. Let $E=\mathbf{R}^{n}$ and choose $E^{\prime}=\mathbf{R}^{n}$. Let $f, g: \mathbf{R}^{n} \rightarrow[-\infty,+\infty]$ be two functions satisfying the hypotheses of the theorem. Assume in addition that $f$ is finite in a neighborhood of the origin and grows faster than any linear function, and that $g$ is not the constant $+\infty$. Then

$$
\operatorname{order}(f: g)=\limsup _{\xi \rightarrow \infty} \frac{\widetilde{g}(\xi)}{\widetilde{f}(\xi)} .
$$

Proof. If $f \leqslant M$ for $|x|<\underset{\tilde{f}}{\varepsilon}$ we obtain $\tilde{f}(\xi) \geqslant \varepsilon|\xi|-M$. Therefore $0<\tilde{f}<+\infty$ in a neighborhood of $\infty$, and $\lim \tilde{f}=+\infty$, so that the type is given by

$$
\operatorname{type}(\widetilde{g}: \widetilde{f})=\limsup _{\xi \rightarrow \infty} \frac{\widetilde{g}(\xi)}{\widetilde{f}(\xi)} \text {. }
$$




\subsection{The infimal convolution}

The infimal convolution is an important operation in convexity theory. It is actually dual to addition, so many problems can be reduced to simple questions using the Fenchel transformation, but it is often preferable to work directly with it. In this section we just recall the definition.

The infimal convolution of two functions $f, g: E \rightarrow[-\infty,+\infty]$ is defined by

$$
f \square g(x)=\inf _{y}(f(y) \dot{+} g(x-y)), \quad x \in E .
$$

Here the sign $\dot{+}$ denotes upper addition; see section 3.3. The Fenchel transform of an infimal convolution is

$$
(f \square g)^{\sim}(\xi)=\widetilde{f}(\xi)+\widetilde{g}(\xi), \quad \xi \in E^{\prime},
$$

where + is lower addition. (It might seem strange that we get lower addition here, for in general $f \dot{+} g$ is convex when both $f$ and $g$ are convex, but not $f+g$. However, in this case $\widetilde{f}+\widetilde{g}$ equals $\widetilde{f} \dot{+} \widetilde{g}$ except when it is constant, so it is always convex.)

The infimal convolution is sometimes called the epigraphical sum. The explanation is the following formula for the strict epigraph (cf. 1.1.4) of $f \square g$

$$
\operatorname{epi}_{s}(f \square g)=\operatorname{epi}_{s} f+\operatorname{epi}_{s} g
$$

where the plus sign denotes vector addition in $\mathbf{R}^{n+1}$.

\subsection{The order of an entire function}

Let $F \in \mathcal{O}\left(\mathbf{C}^{n}\right)$ be an entire function. We shall measure its growth by

$$
f(t)=\sup _{z}\left[\log |F(z)| ; z \in \mathbf{C}^{n},|z| \leqslant e^{t}\right], \quad t \in \mathbf{R} .
$$

Here $|z|$ can be any norm on $\mathbf{C}^{n}$, or even an arbitrary function which is complex homogeneous of degree one and positive on the unit sphere. We shall refer to $f$ as the growth function of $F$. In view of Hadamard's three-circle theorem, $f$ is convex and increasing, and we shall write

$$
\operatorname{order}(F: G)=\operatorname{order}(f: g)
$$

by abuse of language if $F, G$ are two entire functions and $f, g$ are their growth functions.

One may ask which convex increasing functions can appear as growth functions. A necessary condition is that of Hayman [1968]: for a transcendental entire function $F \in \mathcal{O}(\mathbf{C})$, we have

$$
\limsup _{t \rightarrow+\infty} f^{\prime \prime}(t) \geqslant H
$$

where $H$ is an absolute constant satisfying $0.18<H \leqslant 0.25$. Kjellberg [1974] proved that $0.24<H<0.25$. (A similar statement holds for polynomials.) Another necessary condition is as follows. Define

$$
f_{1}(t)=\sup _{j \in \mathbf{N}}(j t-\widetilde{f}(j)), \quad t \in \mathbf{R}
$$


(The epigraph of $f_{1}$ is the smallest polygon which contains the epigraph of $f$ and whose sides have integer slopes.) Then there is a constant $C$ such that

$$
f(t)-C \leqslant f_{1}(t) \leqslant f(t), \quad t \in \mathbf{R} .
$$

Moreover the best constant $C$ satisfies $\log 2 \leqslant C \leqslant \log 3$ (Kiselman [1984, Proposition 3.5.1]). These two results are not unrelated, for the latter implies that $H \geqslant(8 C)^{-1}$. With $C=\log 3$ this gives $H \geqslant(8 \log 3)^{-1} \approx 0.11$, which is much weaker than the Hayman-Kjellberg result. On the other hand, that statement does not say anything about tangents of integer slope.

If two entire functions $F$ and $G$ are given, we consider their expansions in terms of homogeneous polynomials $P_{j}$ and $Q_{j}$ :

$$
F(z)=\sum_{0}^{\infty} P_{j}(z), \quad G(z)=\sum_{0}^{\infty} Q_{j}(z),
$$

and ask whether we can determine $\operatorname{order}(F: G)$ from knowledge of the growth of $\left|P_{j}\right|$ and $\left|Q_{j}\right|$. It turns out that this is so. For the classical order, when $G=\exp$, this is well known. This is not necessarily true for type $(F: G)$; see Kiselman [1993].

So let $F$ be given with an expansion in terms of homogeneous polynomials $P_{j}$. Cauchy's inequalites say that

$$
\left|P_{j}(z)\right| \leqslant \exp (f(\log |z|)),
$$

but the homogeneity of $P_{j}$ also gives

$$
\left|P_{j}(z)\right|=\frac{|z|^{j}}{e^{j t}} P_{j}\left(e^{t} z /|z|\right) \leqslant \frac{|z|^{j}}{e^{j t}} \exp (f(t))=|z|^{j} \exp (f(t)-j t)
$$

for all real $t$ and all $z \in \mathbf{C}^{n}$. We take the infimum over all $t$ and get

$$
\left|P_{j}(z)\right| \leqslant|z|^{j} \exp (-\widetilde{f}(j)) .
$$

We define the norm $\left\|P_{j}\right\|$ of the homogeneous polynomial $P_{j}$ as

$$
\left\|P_{j}\right\|=\sup _{|z| \leqslant 1}\left|P_{j}(z)\right|, \quad j \in \mathbf{N} .
$$

(When $n=1$ we have a Taylor expansion $F(z)=\sum a_{j} z^{j}$ and $\left\|P_{j}\right\|=\left|a_{j}\right|$.) We next define a function $p: \mathbf{R} \rightarrow]-\infty,+\infty]$ as

$$
p(j)= \begin{cases}-\log \left\|P_{j}\right\| & \text { when } j \in \mathbf{N} \\ +\infty & \text { when } j \in \mathbf{R} \backslash \mathbf{N} .\end{cases}
$$

We shall call $p$ the coefficient function of $F$. Cauchy's inequalities become just $\left\|P_{j}\right\| \leqslant$ $\exp (-\tilde{f}(j))$, or more concisely

$$
p \geqslant \tilde{f} \quad \text { on } \mathbf{R} \text {. }
$$

This implies of course that $\widetilde{p} \leqslant \widetilde{\widetilde{f}}=f$. Note also that

$$
\exp \widetilde{p}(\log r)=\sup _{j \in \mathbf{N}} \sup _{|z| \leqslant r}\left|P_{j}(z)\right| .
$$


We now ask for inequalities in the other direction. To describe this result we need an auxiliary function $K$ which is defined as follows:

$$
K(t)= \begin{cases}-\log \left(1-e^{t}\right), & t<0 \\ +\infty, & t \geqslant 0 .\end{cases}
$$

We have $K(t) \geqslant-\log (-t)$ when $t<0$ (a good approximation for small $|t|$ ) and $K(t) \geqslant e^{t}$ for all $t$ (a good approximation for $t \ll 0$ ). The Fenchel transform of $K$ is

$$
\widetilde{K}(\tau)= \begin{cases}\tau \log \tau-(\tau+1) \log (\tau+1), & \tau>0 \\ 0, & \tau=0 \\ +\infty, & \tau<0 .\end{cases}
$$

We note that

$$
-1-\log (\tau+1) \leqslant \widetilde{K}(\tau) \leqslant-\log (\tau+1), \quad \tau \geqslant 0 .
$$

The inverse of $K$ is given by $K^{-1}(s)=-K(-s)$ for $s>0$ : this means that the graph of $K$ is symmetric around the line $s+t=0$. This symmetry corresponds to the functional equation $\widetilde{K}(1 / \tau)=\widetilde{K}(\tau) / \tau, \tau>0$, for the transform.

Theorem 3.6.1. Let $F$ be an entire function in $\mathbf{C}^{n}$ and define $f$ and $p$ by (3.6.1) and (3.6.2), respectively. Then

$$
\widetilde{p} \leqslant f \leqslant \widetilde{p} \square K \quad \text { on } \mathbf{R} .
$$

Proof. We have just noted that Cauchy's inequalities give $\widetilde{p} \leqslant \widetilde{\widetilde{f}}=f$. To estimate $f$ from above we write

$$
|F(z)| \leqslant \sum\left\|P_{j}\right\| \cdot|z|^{j} \leqslant \sum \exp (-p(j)+j t)
$$

where $t=\log |z|$. We shall apply Fenchel's inequality (3.4.3) $j t \leqslant p(j) \dot{+} \widetilde{p}(t)$ in the form

$$
-p(j)+j t \leqslant j s+\widetilde{p}(t-s) .
$$

This gives

$$
f(t) \leqslant \log \sum \exp (-p(j)+j t) \leqslant \log \sum \exp (j s+\widetilde{p}(t-s)) .
$$

We observe that

$$
\sum_{j \in \mathbf{N}} e^{j s}=\frac{1}{1-e^{s}}=e^{K(s)}
$$

if $s<0$, which is why we introduced $K$. Thus $f(t) \leqslant \widetilde{p}(t-s)+K(s)$ for all $t \in \mathbf{R}$ and all $s<0$. Now for $s \geqslant 0, K(s)=+\infty$, so then the inequality also holds, and we can let $s$ vary over the whole real axis:

$$
f(t) \leqslant \inf _{s}(\widetilde{p}(t-s) \dot{+} K(s))=(\widetilde{p} \square K)(t), \quad t \in \mathbf{R} .
$$

This proves the theorem.

The inequalities (3.6.6) say that the graph of $f$ is in a strip whose lower boundary is the polygon defined by $\widetilde{p}$ and whose upper boundary is given by $\widetilde{p} \square K$. Since $K(-\log 2)=\log 2$, the width of this strip is at most $\sqrt{2} \log 2 \approx 0.98<1$.

Applying the Fenchel transformation to all members of (3.6.6) we get:

$$
p \geqslant \tilde{\widetilde{p}} \geqslant \tilde{f} \geqslant \widetilde{\widetilde{p}}+\widetilde{K},
$$

where $\widetilde{K}$ can be estimated by (3.6.5).

For lacunary series we can state: 
Theorem 3.6.2. Let $F$ be lacunary: $P_{j}=0$ for $j \notin J$. Then

$$
\widetilde{p} \leqslant f \leqslant \widetilde{p} \square K_{J} \quad \text { on } \mathbf{R},
$$

where

$$
K_{J}(s)=\log \left(\sum_{j \in J} e^{j s}\right)
$$

Proof. Just restrict summation in the proof of Theorem 3.6.1 to $j \in J$.

It could be noted here that for any convex function $H$ which is positive on the negative half-axis and tends to $+\infty$ as $t<0, t \rightarrow 0$, there exists an infinite set $J \subset \mathbf{N}$ such that $K_{J} \leqslant H$.

Theorem 3.6.1 implies that the norms of the homogeneous polynomials $P_{j}$ can serve just as well as the growth function $f$ to determine the order of $F$ relative to any other function. More precisely we have:

Corollary 3.6.3. Let $F$ be an entire function on $\mathbf{C}^{n}$, let $f$ be its growth function defined by (3.6.1), and let $p$ be its coefficient function defined by (3.6.2). Assume that $F$ is not a polynomial. Then

$$
\operatorname{order}(f: \widetilde{p})=\operatorname{order}(\widetilde{p}: f)=1 \text {. }
$$

Proof. From (3.6.6) we get immediately

$$
\operatorname{order}(\widetilde{p}: f) \leqslant 1, \quad \operatorname{order}(f: \widetilde{p} \square K) \leqslant 1 .
$$

Now $\widetilde{p} \square K(t) \leqslant \widetilde{p}(t+1)+K(-1)$ and Lemma 3.3.2 shows that the translation of $\widetilde{p}$ does not influence the order, neither does of course the additive constant $K(-1)$. Therefore

$$
\operatorname{order}(f: \widetilde{p}) \leqslant \operatorname{order}(f: \widetilde{p} \square K) \leqslant 1 .
$$

It follows from Corollary 3.4.4 that $\operatorname{order}(f: f)=1$. By submultiplicativity,

$$
1=\operatorname{order}(f: f) \leqslant \operatorname{order}(f: \widetilde{p}) \cdot \operatorname{order}(\widetilde{p}: f) \leqslant 1,
$$

so that all orders must be one. (When $F$ is a polynomial, order $(f: f)=0$ and $\operatorname{order}(f: \widetilde{p})=$ $\operatorname{order}(\widetilde{p}: f)=0$.)

Corollary 3.6.4. Let $F$ be an entire function in $\mathbf{C}^{n}$, with expansion

$$
F(z)=\sum P_{j}(z)
$$

in terms of homogeneous polynomials $P_{j}$. Let $f$ be its growth function defined by (3.6.1) and let $p$ be its coefficient function defined by (3.6.2). Let $g: \mathbf{R} \rightarrow[-\infty,+\infty]$ be any function which satisfies $\widetilde{g}=g$. Then

$$
\operatorname{order}(f: g)=\operatorname{order}(\widetilde{p}: g)=\operatorname{type}(\widetilde{g}: \widetilde{\widetilde{p}}) .
$$


When $F$ is not a polynomial and $g$ is bounded from below and not identically $+\infty$, the order is also given by

$$
\limsup _{j \rightarrow+\infty} \frac{\widetilde{g}(j)}{p(j)}
$$

Proof. Using Corollary 3.6.3 we can write

$$
\operatorname{order}(f: g) \leqslant \operatorname{order}(f: \widetilde{p}) \cdot \operatorname{order}(\widetilde{p}: g)=\operatorname{order}(\widetilde{p}: g)
$$

provided $F$ is not a polynomial. Similarly

$$
\operatorname{order}(\widetilde{p}: g) \leqslant \operatorname{order}(\widetilde{p}: f) \cdot \operatorname{order}(f: g)=\operatorname{order}(f: g) \text {. }
$$

The last equality in (3.6.8) follows from Theorem 3.4.3. If $F$ is a polynomial, one can verify (3.6.8) directly, using $\widetilde{p} \leqslant f \leqslant \widetilde{p}+\log N$, where $N$ is the number of terms in the expansion (see Theorem 3.6.2). The only possibilities are then $\operatorname{order}(f: g)=0,+\infty$.

We finally have, if $F$ is not a polynomial and $g$ is bounded from below and not identically $+\infty$

$$
\operatorname{type}(\widetilde{g}: \widetilde{\widetilde{p}})=\limsup _{\tau \rightarrow+\infty} \frac{\widetilde{g}(\tau)}{\widetilde{\widetilde{p}}(\tau)}=\limsup _{j \rightarrow+\infty} \frac{\widetilde{g}(j)}{p(j)} .
$$

The first equality here is proved like in the proof of Corollary 3.4.4. There is a difference in that $f(t)$ does not go to $+\infty$ when $t \rightarrow-\infty$, but if $g$ is bounded from below, the behavior for negative $\tau$ in (3.6.10) is unimportant. The last equality in (3.6.10) holds because on the one hand $\widetilde{\widetilde{p}} \leqslant p$, on the other hand $\widetilde{\widetilde{p}}=p$ in a sequence of integers tending to plus infinity, and $\widetilde{\widetilde{p}}$ is affine in between these points.

Formula (3.6.9) generalizes the classical formula for the order

$$
\rho=\limsup \frac{j \log j}{-\log \left|a_{j}\right|}
$$

of an entire function $\sum a_{j} z^{j}$. Indeed, when the comparison function is $g(t)=e^{t}$, then $\widetilde{g}(j)=j \log j-j$.

The $(p, q)$-order of Juneja, Kapoor \& Bajpai [1976, Theorem 1] is determined in terms of the coefficients by the formula

$$
\rho_{p, q}=\limsup \frac{\log ^{[p-1]} j}{\log ^{[q-1]}\left(-(1 / j) \log \left|a_{j}\right|\right)} ;
$$

we state it only for $p>q \geqslant 1$ here. Sato [1963] proved this for $q=1$. In the latter case (3.6.9) is a generalization. For $q \geqslant 2$, however, this is not so, since then $f\left(\exp ^{[q-1]} t\right)$ is used as the growth function and consequently defines another relation between the coefficients $a_{j}$ (or $p(j)$ ) and $f$.

It could also be noted here that Corollary 3.6.4 generalizes the classical result that the order can be calculated from the dominant term in a series expansion $\sum a_{j} z^{j}$. Indeed, with $t=\log |z|$ the maximal term is just

$$
\sup _{j}\left|a_{j} z^{j}\right|=\exp \sup _{j}(j t-p(j))=\exp \widetilde{p}(t) .
$$

When we have two entire functions we can state: 
Corollary 3.6.5. Let $F, G$ be two entire functions in $\mathbf{C}^{n}$, with expansions

$$
F(z)=\sum P_{j}(z), \quad G(z)=\sum Q_{j}(z),
$$

in terms of homogeneous polynomials $P_{j}, Q_{j}$. Let $p$ and $q$ denote their coefficient functions defined by (3.6.2). Then

$$
\operatorname{order}(F: G)=\operatorname{order}(\widetilde{p}: \widetilde{q})=\operatorname{type}(\widetilde{\widetilde{q}}: \widetilde{\widetilde{p}}) .
$$

Proof. The proof is analogous to that of Corollary 3.6.4.

We can also define a growth function related to the growth of an entire function on polydisks, and to Taylor expansions in terms of monomials. Let us define

$$
f(x)=\sup _{\left|z_{j}\right| \leqslant \exp x_{j}} \log |F(z)|, \quad x \in \mathbf{R}^{n},
$$

if $F$ is an entire function on $\mathbf{C}^{n}$. Then $f$ is convex in $\mathbf{R}^{n}$. The function $F$ has an expansion

$$
F(z)=\sum_{k \in \mathbf{N}^{n}} A_{k} z^{k}, \quad z \in \mathbf{C}^{n}
$$

where $z^{k}$ denotes the monomial $z_{1}^{k_{1}} \cdots z_{n}^{k_{n}}$ of multidegree $k=\left(k_{1}, \ldots, k_{n}\right)$ and total degree $k_{1}+\cdots+k_{n}$. Cauchy's inequalities now say that, for $r=\left(r_{1}, \ldots, r_{n}\right)$ with $r_{j}>0$,

$$
\left|A_{k}\right| r^{k} \leqslant \sup _{\left|z_{j}\right| \leqslant r_{j}}|F(z)|=e^{f(x)}, \quad x_{j}=\log r_{j} .
$$

This gives $\left|A_{k}\right| \leqslant \exp (f(x)-k \cdot x)$ for all $x \in \mathbf{R}^{n}$, and therefore, after variation of $x$,

$$
\left|A_{k}\right| \leqslant \exp (-\widetilde{f}(k)), \quad k \in \mathbf{N}^{n} .
$$

We introduce in analogy with (3.6.2)

$$
a(k)= \begin{cases}-\log \left|A_{k}\right| & \text { when } k \in \mathbf{N}^{n} \\ +\infty & \text { when } k \in \mathbf{R}^{n} \backslash \mathbf{N}^{n} .\end{cases}
$$

Then $a \geqslant \tilde{f}$ and $\widetilde{a} \leqslant \widetilde{\widetilde{f}}=f$. Next define $K_{n}(x)=K\left(x_{1}\right)+\cdots+K\left(x_{n}\right)$ for $x \in \mathbf{R}^{n}$. In complete analogy with Theorem 3.6.1 we have:

Theorem 3.6.6. Let $F$ be an entire function in $\mathbf{C}^{n}$ and define the growth function $f$ and the coefficient function a by (3.6.11) and (3.6.12), respectively. Then

$$
\widetilde{a} \leqslant f \leqslant \widetilde{a} \square K_{n} \quad \text { on } \mathbf{R}^{n} .
$$

A variant of the growth function can be defined as follows. Let $u$ be a plurisubharmonic function on $\mathbf{C}^{n}$ which is extremal in the set $a<u(z)<b$ : it is the regularized supremum of all plurisubharmonic functions $\varphi$ in a neighborhood of the closure of $\{z ; a<u(z)<b\}$ which 
satisfy $\varphi(z) \leqslant a$ when $u(z) \leqslant a$ and $\varphi(z) \leqslant b$ when $u(z) \leqslant b$. We suppose that $\{z ; u(z)<b\}$ is bounded, and define for $F \in \mathcal{O}\left(\mathbf{C}^{n}\right)$

$$
f_{u}(t)=\sup _{z}(\log |F(z)| ; u(z)<t) .
$$

Then $f_{u}$ is easily seen to be convex on $\left.] a, b\right]$. (The growth function $f$ defined by (3.6.1) is with respect to the extremal plurisubharmonic function $u(z)=\log |z|$ provided $|z|$ is a norm or more generally $\log |z|$ is plurisubharmonic; if not, we can replace it by a suitable plurisubharmonic minorant.)

We can for instance ask whether a holomorphic function on a complex analytic variety $X$ admits an entire extension of the same order: if $F \in \mathcal{O}(X), X \subset \mathbf{C}^{n}$, does there exist an entire function $G \in \mathcal{O}\left(\mathbf{C}^{n}\right)$ such that $\operatorname{order}(G: F)=1$ ? Here it might be natural to define the growth functions $f_{u}$ and $g_{v}$ of $F$ and $G$ with respect to extremal functions $u$ on $X$ and $v$ on $\mathbf{C}^{n}$, respectively.

\subsection{A geometric characterization of the relative order}

In this section we shall give a geometric interpretation of the relative order. Let $E$ be a real vector space. We consider two hyperplanes $E \times\{0\}$ and $E \times\{1\}$ in the Cartesian product $E \times \mathbf{R}$. Now let two functions $\left.\left.f_{0}, f_{1}: E \rightarrow\right]-\infty,+\infty\right]$ be given. We consider them as defined on $E \times\{0\}$ and $E \times\{1\}$ respectively, and want to find a function $F: E \times \mathbf{R} \rightarrow]-\infty,+\infty]$ extending them, i.e., a function such that

$$
F(x, j)=f_{j}(x), \quad x \in E, \quad j=0,1 .
$$

If the $f_{j}$ are convex, a solution is of course the supremum of all convex minorants to the function $f(x, t)=f_{t}(x)$ if $t=0$ or $t=1, f(x, t)=+\infty$ otherwise. This solution is the largest possible: it majorizes all others. But it is of no interest outside the slab $\{0 \leqslant t \leqslant 1\}$, since it is always $+\infty$ there.

In general there is no unique solution, for we can always add $t^{2}-t$ to any given solution. We can however write down an explicit formula for an extremal solution.

Proposition 3.7.1. Let $E$ be a real vector space and $E^{\prime}$ a subspace of its algebraic dual. Let $\left.\left.f_{0}, f_{1}: E \rightarrow\right]-\infty,+\infty\right]$ be two given convex functions which are lower semicontinuous with respect to $\sigma\left(E, E^{\prime}\right)$. We assume that they are not identically plus infinity. Then the extrapolation problem

$$
\left\{\begin{array}{l}
\text { Find } F: E \times \mathbf{R} \rightarrow]-\infty,+\infty] \text { such that } \\
F(x, j)=f_{j}(x), \quad x \in E, \quad j=0,1
\end{array}\right.
$$

has a solution

$$
\begin{aligned}
F(x, t) & =\sup _{\xi}\left[\xi \cdot x-(1-t) \widetilde{f}_{0}(\xi)-t \widetilde{f}_{1}(\xi) ; \xi \in \operatorname{dom} \widetilde{f}_{0} \cup \operatorname{dom} \widetilde{f}_{1}\right] \\
& =\sup _{\xi}\left[\xi \cdot x-\left((1-t) \widetilde{f}_{0}(\xi) \dot{+} t \widetilde{f}_{1}(\xi)\right) ; \xi \in E^{\prime}\right], \quad(x, t) \in E \times \mathbf{R} .
\end{aligned}
$$

This solution is extremal in the sense that any convex solution $G$ which is lower semicontinuous in $x$ satisfies $G \leqslant F$ in $\{0 \leqslant t \leqslant 1\}$ and $G \geqslant F$ outside this slab. 
Proof. First a word about the definition of $F$. We note that the function $t \mapsto t \cdot(+\infty)$ is convex on the whole real line, if we define $0 \cdot(+\infty)=0$. We also note that in the first expression defining $F$ at most one of the three terms is infinite, for we have $-\infty<\widetilde{f}_{j} \leqslant+\infty$ everywhere, and at most one of them is allowed to be plus infinity in the set of $\xi$ which we use. Therefore $F$ is well defined, and it is convex as a supremum of functions of $(x, t)$ each of which is an affine function plus possibly one function of the form $(t-1) \cdot(+\infty)$ or $(-t) \cdot(+\infty)$. Moreover, for $t=j$ the function $F$ assumes the values $\widetilde{\widetilde{f}}_{j}(x)=f_{j}(x), j=0,1$, in view of (3.4.2). Therefore it is a convex solution to the extension problem. It is of course not lower semicontinuous in all variables, but it is lower semicontinuous in $x$ for fixed $t$.

Now let $G$ be another convex solution to the problem. Let us consider

$$
\widetilde{G}_{t}(\xi)=\sup _{x \in E}(\xi \cdot x-G(x, t)), \quad t \in \mathbf{R}, \quad \xi \in E^{\prime} .
$$

It is concave in $t$ for fixed $\xi$, for it is the marginal function of a concave function of $(x, t)$; cf. Theorem 1.3.1. It satisfies moreover $\widetilde{G}_{j}(\xi)=\widetilde{f}_{j}(\xi), j=0,1$. If we assume that $G$ is lower semicontinuous in $x$ and $>-\infty$, we also have

$$
G(x, t)=\sup _{\xi}\left(\xi \cdot x-\widetilde{G}_{t}(\xi)\right) .
$$

When $0<t<1$ we have

$$
\operatorname{dom}\left((1-t) \widetilde{f}_{0}+t \widetilde{f}_{1}\right)=\operatorname{dom} \widetilde{f}_{0} \cap \operatorname{dom} \widetilde{f}_{1} \subset \operatorname{dom} \tilde{f}_{0} \cup \operatorname{dom} \tilde{f}_{1}
$$

The fact that $\widetilde{G}_{j}=\widetilde{f}_{j}$ for $j=0,1$ implies that $\widetilde{G}_{t} \geqslant(1-t) \widetilde{f}_{0}+t \widetilde{f}_{1}$. This gives $G \leqslant F$.

When $t<0$ or $t>1$ the concavity in $t$ gives $\widetilde{G}_{t} \leqslant(1-t) \widetilde{f}_{0}+t \widetilde{f}_{1}$ and then $G \geqslant F$. This establishes the extremal character of the solution $F$.

We now ask how far outside the slab $\{0 \leqslant t \leqslant 1\}$ we can obtain a real-valued solution to the extrapolation problem. An answer is given by the next theorem.

Theorem 3.7.2. Let $\left.\left.f_{0}, f_{1}: E \rightarrow\right]-\infty,+\infty\right]$ be two given convex and lower semicontinuous functions. Assume that $f_{0}(0)<+\infty$. If the extrapolation problem (3.7.1) admits a convex solution $F$ which is finite at a point $(0, t)$ with $t$ satisfying $1<t<+\infty$, then

$$
\operatorname{order}\left(f_{1}: f_{0}\right) \leqslant \frac{t}{t-1}
$$

Conversely, if $1 \leqslant \operatorname{order}\left(f_{1}, f_{0}\right)=\rho<+\infty$, then the extrapolation problem has a lower semicontinuous convex solution $F$ with $F(0, t)<+\infty$ for all $t$ with $0 \leqslant t<\rho /(\rho-1)$. Thus if we denote by $b$ the supremum of all numbers $t$ such that there exists a solution $F$ which is finite at the point $(0, t)$, then

$$
\operatorname{order}\left(f_{1}: f_{0}\right)=\rho=\frac{b}{b-1}=b^{\prime} .
$$

(We assume $1 \leqslant \rho<+\infty$ and $1<b \leqslant+\infty$.) 
Proof. If $F$ is convex we have

$$
F(x, 1) \leqslant \frac{1}{a} F(a x, 0) \dot{+}\left(1-\frac{1}{a}\right) F(0, t),
$$

where $a>1$ is chosen so that

$$
(x, 1)=\frac{1}{a}(a x, 0)+\left(1-\frac{1}{a}\right)(0, t) \in E \times \mathbf{R},
$$

i.e., $a=t /(t-1)$. Now if $F(0, t)<+\infty$ this inequality shows that

$$
f_{1}(x) \leqslant \frac{1}{a} f_{0}(a x)+c
$$

in other words that $\operatorname{order}\left(f_{1}: f_{0}\right) \leqslant a=t /(t-1)$.

Conversely, if $\operatorname{order}\left(f_{1}, f_{0}\right) \leqslant \rho$ with $1 \leqslant \rho<+\infty$, then the solution $F$ defined by (3.7.2) has the desired properties. We need only estimate $F$ as follows. For any $a>\rho$ we know that $f_{1}(x) \leqslant f_{0}(a x) / a+c$, which gives $\widetilde{f}_{1} \geqslant a^{-1} \widetilde{f}_{0}-c$. In particular we see that $\operatorname{dom} \widetilde{f}_{0} \supset \operatorname{dom} \widetilde{f}_{1}$. For any $t<a /(a-1)$ we can write, letting $\xi$ vary in $\operatorname{dom} \widetilde{f}_{0}$,

$$
\begin{aligned}
F(x, t) & \leqslant \sup _{\xi}\left[\xi \cdot x-(1-t) \widetilde{f}_{0}(\xi)-t\left(a^{-1} \widetilde{f}_{0}(\xi)-c\right)\right]=\sup _{\xi}\left[\xi \cdot x-(1-t+t / a) \widetilde{f}_{0}(\xi)\right]+t c \\
& =(1-t+t / a) \sup _{\xi}\left[(1-t+t / a)^{-1} \xi \cdot x-\widetilde{f}_{0}(\xi)\right]+t c=\delta f_{0}(x / \delta)+t c,
\end{aligned}
$$

where $\delta$ is the positive number $1-t+t / a$. Now, since we assume that $f_{0}(0)<+\infty$, this shows that $F(0, t)$ is finite for all $t \in[0, a /(a-1)$ [, and since $a$ is any number larger than $\rho$, the function is finite for all $t \in[0, b[$.

For real-valued functions the geometry is particularly simple:

Corollary 3.7.3. Let $\left.\left.f_{0}, f_{1}: E \rightarrow\right]-\infty,+\infty\right]$ be two functions as in Proposition 3.7.1 and assume in addition that one of them is real valued. If the extrapolation problem (3.7.1) admits a convex solution $F$ which is finite at some point $(x, t)$ with $t$ satisfying $1<t<+\infty$, then

$$
\operatorname{order}\left(f_{1}: f_{0}\right) \leqslant \frac{t}{t-1}
$$

Conversely, if $1 \leqslant \operatorname{order}\left(f_{1}, f_{0}\right)=\rho<+\infty$, then the extrapolation problem has a lower semicontinuous convex solution $F$ which is real valued in the slab

$$
E \times] 0, \rho^{\prime}\left[=\left\{(x, t) \in E \times \mathbf{R} ; 0<t<\rho^{\prime}\right\},\right.
$$

where $\rho^{\prime}=\rho /(\rho-1) ; 1<\rho^{\prime} \leqslant+\infty$.

Therefore the relative order of $f_{1}$ with respect to $f_{0}$ is determined by, and determines, the maximal slab $E \times] 0, b[$ in which our extrapolation problem has a solution.

Proof. Suppose $f_{j}$ is real valued $(j=0$ or $j=1)$. It is clear that if a solution $F$ is finite at some point $(x, s)$ with $s>1$, then $F$ is finite in the convex hull of the union of $(x, s)$, some 
point $(y, 0)$ where $f_{0}$ is finite, and the hyperplane $E \times\{j\}$. This convex hull contains the slab $E \times] 0, s[$. Thus Theorem 3.7.2 implies Corollary 3.7.3.

It follows again (cf. Lemma 3.3.2) that the notion of relative order is translation invariant for real-valued convex functions (at least when $1 \leqslant \rho+\infty$ ). Indeed, the slabs are invariant under transformations $(x, t) \mapsto(x-(1-t) y-t z, t)$ for all $y$ and $z$; these transformations correspond to translations $f_{0} \mapsto f_{0, y}$ and $f_{1} \mapsto f_{1, z}$.

\subsection{An extension theorem for holomorphic functions}

In this section we shall first characterize the classical order in terms of an extension property of holomorphic functions. Then we pass to the relative order.

Theorem 3.8.1. An entire function $F \in \mathcal{O}\left(\mathbf{C}^{n}\right)$ is of order at most $\rho(1 \leqslant \rho<+\infty)$ if and only if there exists a holomorphic function $H$ in the cylinder

$$
\Omega=\left\{(z, w) \in \mathbf{C}^{n} \times \mathbf{C} ;|w|<e^{\rho^{\prime}}\right\}
$$

where $\rho^{\prime}=\rho /(\rho-1)$, satisfying

$$
|H(z, w)| \leqslant e^{|z|} \quad \text { for } z \in \mathbf{C}^{n},|w| \leqslant 1,
$$

and

$$
H(z, e)=F(z) \quad \text { for } z \in \mathbf{C}^{n}
$$

Proof. Suppose such an $H$ exists. Then putting

$$
h(s, t)=\sup \left[\log |H(z, w)| ;|z| \leqslant e^{s},|w| \leqslant e^{t}\right], \quad s \in \mathbf{R}, t<\rho^{\prime}
$$

we get a convex function of $(s, t)$ which satisfies $h(s, 0) \leqslant e^{s}$ and $h(s, 1) \geqslant f(s)$. Therefore, applying Corollary 3.7.3 with $f_{0}(s)=h(s, 0)$ and $f_{1}(s)=h(s, 1)$, we can write

$$
\operatorname{order}(F: \exp ) \leqslant \operatorname{order}\left(f_{1}: \exp \right) \leqslant \operatorname{order}\left(f_{1}: f_{0}\right) \cdot \operatorname{order}\left(f_{0}: \exp \right) \leqslant \rho .
$$

In the other direction the results of section 3.7 give only convex, not holomorphic, solutions to the extrapolation problem. But it turns out that there is an explicit solution in terms of power series.

We expand $F$ in a series of homogeneous polynomials:

$$
F(z)=\sum P_{j}(z)
$$

Then we just define

$$
H(z, w)=\sum P_{j}(z)(w / e)^{m_{j}}
$$

where the integers $m_{j}$ are chosen large enough to make (3.8.1) true. This means that we take

$$
\left\|P_{j}\right\| e^{-m_{j}} \leqslant \frac{1}{j !}
$$


On the other hand, we do not want to take them unnecessarily large, so we prescribe that

$$
\log \left\|P_{j}\right\|+\log j ! \leqslant m_{j}<\log \left\|P_{j}\right\|+\log j !+1
$$

unless $P_{j}=0$ in which case the choice of $m_{j}$ is immaterial, so we may take $m_{j}=0$.

Since $F$ is of order $\rho$, we know that for any $a>\rho$ there is an estimate $f(t) \leqslant e^{a t}+C_{a}$, which implies that

$$
\widetilde{f}(\tau) \geqslant \frac{\tau}{a}\left(\log \frac{\tau}{a}-1\right)-C_{a}
$$

and

$$
-\log \left\|P_{j}\right\|=p(j) \geqslant \widetilde{f}(j) \geqslant \frac{j}{a}\left(\log \frac{j}{a}-1\right)-C_{a} .
$$

This estimate shows that the series defining $H$ converges uniformly on any compact subset of $\Omega$. In fact, the series defining $H$ converges uniformly for $|z| \leqslant R_{1}<R$ and $|w| \leqslant r_{1}<$ $r$ if $\left\|P_{j}\right\| R^{j}(r / e)^{m_{j}} \rightarrow 0$. Substituting the expression for $m_{j}$ we see that this is true if $(\log r-1) \log j !-p(j) \log r+j \log R \rightarrow-\infty$. Now this holds for all positive $R$ if

$$
\frac{(\log r-1) \log j !-p(j) \log r}{j} \rightarrow-\infty .
$$

Using finally the estimate (3.8.5) for $p$ and the inequality $j ! \leqslant j^{j}$ for the factorial function we see that this follows if

$$
(\log r-1) \log j-\frac{1}{a}\left(\log \frac{j}{a}-1\right) \log r \rightarrow-\infty,
$$

which in turn is true if $\log r<a /(a-1)$. Here the only condition is that $a>\rho$, so the series defining $H$ converges locally uniformly in the set $\log |w|<\rho /(\rho-1)$.

We now replace the exponential function in Theorem 3.8.1 to obtain the follwong result.

Theorem 3.8.2. Let two transcendental entire functions $F, G \in \mathcal{O}\left(\mathbf{C}^{n}\right)$ be given, and let $1 \leqslant \rho<+\infty$. We define an open set $\Omega$ in the space of $n+1$ variables as

$$
\Omega=\left\{(z, w) \in \mathbf{C}^{n} \times \mathbf{C} ;|w|<e^{\rho^{\prime}}\right\}
$$

where $\rho^{\prime}=\rho /(\rho-1)$ (thus $\left.1<\rho^{\prime} \leqslant+\infty\right)$. For a holomorphic function $H$ in $\Omega$ we denote by $h_{w}$ the growth function of the partial function $z \mapsto H(z, w)$. Let $K$ denote the function defined by (3.6.4). Then the following five conditions are equivalent.

(a) $\operatorname{order}(F: G) \leqslant \rho$.

(b) There exists a holomorphic function $H \in \mathcal{O}(\Omega)$ satisfying

$$
h_{w} \leqslant g \square K \quad \text { when }|w|=1, \quad \text { and } \quad f \leqslant h_{w} \square K \quad \text { when }|w|=e .
$$

$\left(\mathrm{b}^{\prime}\right)$ There exists a holomorphic function $H \in \mathcal{O}(\Omega)$ satisfying $H(z, 1)=G(z)$,

$$
g \leqslant h_{w} \square K, \quad \text { and } \quad h_{w} \leqslant g \square K \quad \text { when }|w|=1,
$$


and

$$
f \leqslant h_{w} \square K \quad \text { when }|w|=e .
$$

(c) There exists a holomorphic function $H \in \mathcal{O}(\Omega)$ satisfying $H(z, e)=F(z)$ and

$$
h_{w} \leqslant g \square K \quad \text { when }|w|=1 .
$$

$\left(\mathrm{c}^{\prime}\right)$ There exists a holomorphic function $H \in \mathcal{O}(\Omega)$ satisfying $H(z, e)=F(z)$,

$$
f \leqslant h_{w} \square K \quad \text { and } \quad h_{w} \leqslant f \square K \quad \text { when }|w|=e,
$$

and

$$
h_{w} \leqslant g \square K \quad \text { when }|w|=1 .
$$

In particular, $\operatorname{order}(H(\cdot, w): G) \leqslant 1$ for $|w|=1$ and $\operatorname{order}(F: H(\cdot, w)) \leqslant 1$ for $|w|=e$ if $H$ is the holomorphic function whose existence is guaranteed by $(b),\left(b^{\prime}\right)$ or $\left(c^{\prime}\right)$.

Proof. The proof that (b) implies (a) and that (c) implies (a) is just like the easy direction in the proof of Theorem 3.8.1. If $H$ is a holomorphic function satisfying (b) or (c) we let $h$ be the growth function of two real variables defined by (3.8.3); it is related to the $h_{w}$ by the formula $h(s, t)=\sup _{|w|=e^{t}} h_{w}(s)$. By submultiplicativity we then have

$$
\operatorname{order}(f: g) \leqslant \operatorname{order}(f: h(\cdot, 1)) \cdot \operatorname{order}(h(\cdot, 1): h(\cdot, 0)) \cdot \operatorname{order}(h(\cdot, 0): g) \leqslant \rho \text {. }
$$

It is also clear that $\left(\mathrm{b}^{\prime}\right)$ implies $(\mathrm{b})$ and that $\left(\mathrm{c}^{\prime}\right)$ implies $(\mathrm{c})$.

For the proof of (a) implies ( $\left.\mathrm{b}^{\prime}\right)$ we expand $G$ and $F$ in terms of homogeneous polynomials:

$$
F(z)=\sum_{j \in \mathbf{N}} P_{j}(z), \quad G(z)=\sum_{j \in \mathbf{N}} Q_{j}(z),
$$

and define

$$
H(z, w)=\sum_{j \in \mathbf{N}} Q_{j}(z) w^{n_{j}}+(w-1) \sum_{j \notin J} Q_{j}^{*}(z) w^{n_{j}}
$$

where $J$ is the set of all $j \in \mathbf{N}$ such that $q(j) \leqslant \widetilde{\widetilde{q}}(j)+\log 3, q$ being the coefficient function of $G$ defined by (3.6.2). Moreover $n_{j}$ are suitable integers and $Q_{j}^{*}$ homogeneous polynomials of degree $j$ and norm $\left\|Q_{j}^{*}\right\|=\frac{1}{3} \exp (-\widetilde{\widetilde{q}}(j)) \geqslant\left\|Q_{j}\right\|$. Let $p_{w}$ denote the coefficient function of the entire function $H(\cdot, w)$. Consider first $|w|=1$ : when $j \in J$ we have $p_{w}(j)=q(j)$, and when $j \notin J$ we can estimate as follows:

$$
\left\|Q_{j} w^{n_{j}}+(w-1) Q_{j}^{*} w^{n_{j}}\right\| \leqslant\left\|Q_{j}\right\|+2\left\|Q_{j}^{*}\right\| \leqslant 3\left\|Q_{j}^{*}\right\|=\exp (-\widetilde{\widetilde{q}}(j)),
$$

so that $p_{w}(j) \geqslant \widetilde{\widetilde{q}}(j)$ when $j \notin J$. Therefore we have $p_{w} \geqslant \widetilde{\widetilde{q}}$ everywhere, and $p_{w}=q$ in $J$, which implies $\widetilde{p}_{w}=\widetilde{q}$ for $|w|=1$. Thus in view of Theorem 3.6.1,

$$
h_{w} \leqslant \widetilde{p}_{w} \square K=\widetilde{q} \square K \leqslant g \square K \quad \text { as well as } \quad g \leqslant \widetilde{q} \square K=\widetilde{p}_{w} \square K \leqslant h_{w} \square K \text {. }
$$


This far the numbers $n_{j}$ play no role; we shall now choose them to get the right kind of growth of $H(\cdot, w)$ for $|w|=e$. When $|w|=e$ and $j \in J$ we have $p_{w}(j)=q(j)-n_{j} \leqslant \widetilde{q}(j)-n_{j}+\log 3$. The homogeneous part of degree $j \notin J$ in $H(z, w)$ can be estimated as

$$
\begin{aligned}
\left\|Q_{j} w^{n_{j}}+(w-1) Q_{j}^{*} w^{n_{j}}\right\| \geqslant e^{n_{j}}\left(\left\|(w-1) Q_{j}^{*}\right\|-\left\|Q_{j}\right\|\right) & \\
& \geqslant e^{n_{j}}(e-2)\left\|Q_{j}^{*}\right\|=\frac{e-2}{3} \exp \left(n_{j}-\widetilde{\widetilde{q}}(j)\right),
\end{aligned}
$$

which gives

$$
\begin{aligned}
& p_{w}(j)=q(j)-n_{j} \leqslant \widetilde{\widetilde{q}}(j)-n_{j}+\log 3 \leqslant \widetilde{\widetilde{q}}(j)-n_{j}+2, \quad j \in J, \\
& p_{w}(j) \leqslant \widetilde{\widetilde{q}}(j)-n_{j}-\log \left(\frac{e-2}{3}\right) \leqslant \widetilde{\widetilde{q}}(j)-n_{j}+2, \quad j \notin J .
\end{aligned}
$$

We shall now choose the integers $n_{j}$ as follows. If $\widetilde{\widetilde{q}}(j)=+\infty$ (this can happen for finitely many numbers $j$ only), then also $\widetilde{\widetilde{p}}(j)=+\infty$ and we choose $n_{j}=0$. If $\widetilde{q}(j)<+\infty$, we choose $n_{j}$ as the smallest non-negative integer which is $\geqslant \widetilde{\widetilde{q}}(j)-\widetilde{\widetilde{p}}(j)+2$. Thus in all cases $p_{w} \leqslant \widetilde{\widetilde{p}}$ for every $w$ with $|w|=e$, so that $\widetilde{p} \leqslant \widetilde{p}_{w}$ and we get

$$
f \leqslant \widetilde{p} \square K \leqslant \widetilde{p}_{w} \square K \leqslant h_{w} \square K, \quad|w|=e .
$$

Finally we have to make sure that $H$ is holomorphic in all of $\Omega$. To prove this it is enough to prove that

$$
\left\|Q_{j}\right\| R^{j} r^{n_{j}} \rightarrow 0 \quad \text { and } \quad\left\|Q_{j}^{*}\right\| R^{j} r^{n_{j}} \rightarrow 0
$$

as $j \rightarrow \infty$ for all $R$ and all $r<e^{\rho^{\prime}}$. This in turn follows if we can prove that

$$
\frac{n_{j} \log r-q(j)}{j} \rightarrow-\infty \quad \text { and } \quad \frac{n_{j} \log r-q^{*}(j)}{j} \rightarrow-\infty
$$

We shall use the fact that type $(\widetilde{\widetilde{q}}: \widetilde{\widetilde{p}})=\operatorname{order}(f: g) \leqslant \rho$, which yields an inequality $\widetilde{\widetilde{q}} \leqslant$ $a \widetilde{\widetilde{p}}+C_{a}$ for every $a>\rho$. If $n_{j}=0$, the first expression in $(3.8 .6)$ is at most $-\widetilde{\widetilde{q}}(j) / j$ which certainly tends to $-\infty$. If $n_{j}>0$, it can be estimated by (it suffices to consider $r>1$ )

$$
\frac{n_{j} \log r-q(j)}{j} \leqslant \frac{(\widetilde{\widetilde{q}}(j)-\widetilde{\widetilde{p}}(j)+3) \log r-\widetilde{\widetilde{q}}(j)}{j} \leqslant \frac{\widetilde{\widetilde{p}}(j)(a \log r-\log r-a)+O(1)}{j},
$$

which tends to $-\infty$ if $\log r<a /(a-1)$.

If $n_{j}=0$, the second expression in (3.8.6) is $-q^{*}(j) / j=-(\widetilde{\widetilde{q}}(j)-\log 3) / j$ which tends to $-\infty$; if $n_{j}>0$, it can be estimated by

$$
\begin{aligned}
\frac{n_{j} \log r-q^{*}(j)}{j} & \leqslant \frac{(\widetilde{\widetilde{q}}(j)-\widetilde{\widetilde{p}}(j)+3) \log r-\widetilde{\widetilde{q}}(j)+\log 3}{j} \\
& \leqslant \frac{\widetilde{\widetilde{p}}(j)(a \log r-\log r-a)+O(1)}{j},
\end{aligned}
$$


which tends to $-\infty$ as soon as $\log r<a /(a-1)$; here again $a$ is any number greater than $\rho$. This proves that the series defining $H$ converges locally uniformly in $\Omega$ and finishes the proof of $\left(b^{\prime}\right)$.

The proof that (a) implies $\left(\mathrm{c}^{\prime}\right)$ is similar to that of Theorem 3.8.1. As in that proof we define $H$ by (3.8.4):

$$
H(z, w)=\sum_{j \in \mathbf{N}} P_{j}(z)(w / e)^{m_{j}}
$$

where we shall choose integers $m_{j}$. Then obviously $H(z, e)=F(z)$. For $|w|=e$ we have $p_{w}(j)=p(j)$. This gives $\widetilde{p}_{w}=\widetilde{p}$ and therefore, for all $w$ with $|w|=e$,

$$
h_{w} \leqslant \widetilde{p}_{w} \square K=\widetilde{p} \square K \leqslant f \square K \quad \text { as well as } \quad f \leqslant \widetilde{p} \square K=\widetilde{p}_{w} \square K \leqslant h_{w} \square K .
$$

For $|w|=1$, on the other hand, we obtain

$$
\left\|P_{j}(w / e)^{m_{j}}\right\|=\exp \left(-m_{j}-p(j)\right) \leqslant \exp \left(-m_{j}-\widetilde{\widetilde{p}}(j)\right) .
$$

Thus, when $|w|=1$ we have $p_{w}(j)=p(j)+m_{j} \geqslant \widetilde{\widetilde{p}}(j)+m_{j}$. We now choose $m_{j}$ so that $p_{w} \geqslant \widetilde{\widetilde{q}}$, which implies $\widetilde{p}_{w} \leqslant \widetilde{q}$ and yields the estimate

$$
h_{w} \leqslant \widetilde{p}_{w} \square K \leqslant \widetilde{q} \square K \leqslant g \square K .
$$

To be explicit, if $\widetilde{\widetilde{q}}(j)=+\infty$, then $\widetilde{\widetilde{p}}(j)=+\infty$ and we take $m_{j}=0$; if $\widetilde{\widetilde{q}}(j)<+\infty$, we take $m_{j}$ as the smallest non-negative integer greater than or equal to $\widetilde{\widetilde{q}}(j)-\widetilde{\widetilde{p}}(j)$. This guarantees that $p_{w} \geqslant \widetilde{\widetilde{q}}$ and gives the estimate above. On the other hand, $m_{j}$ is not too large, which will ensure that $\left\|P_{j}\right\| R^{j}(r / e)^{m_{j}}$ tends to zero for every $R$ and every $r<e^{\rho^{\prime}}$ and hence that $H$ is holomorphic in $\Omega$. The calculation is very similar to the one we just carried out in the case of $\left(\mathrm{b}^{\prime}\right)$ and is omitted. 


\section{References}

Abrahamsson, Leif

1988 Microlocal Lelong numbers of plurisubharmonic functions. J. Reine Angew. Math. 388, 116-128.

Demailly, Jean-Pierre

1987 Nombres de Lelong généralisés, théorèmes d'intégralité et d'analyticité. Acta Math. 159, 153-169.

1989 Potential theory in several complex variables. Lecture notes from the École d'été d'analyse complexe, organized by CIMPA, Nice, July 1989.

Freund, M., and Grlich, E.

1985 On the relation between maximum modulus, maximum term, and Taylor coefficients of an entire function. J. Approx. Thy 43, 194-203.

Halvarsson, Stefan

forthc. Extension of entire functions with controlled growth. Math. Scand. [to appear].

forthc. Growth properties of entire functions depending on a parameter. Manuscript, 20 pages. Uppsala University 1993.

Hayman, W. K.

1968 Note on Hadamard's convexity theorem. Entire functions and related parts of analysis. Proceedings of Symposia in Pure Mathematics, volume 11, 210-213. Providence: American Mathematical Society.

Hörmander, Lars

1990 Complex analysis in several variables. North-Holland.

forthc. Notions of convexity. [Monograph to appear.]

Juneja, O. P., Kapoor, G. P., and Bajpai, S. K.

1976 On the $(p, q)$-order and lower $(p, q)$-order of an entire function. J. Reine Angew. Math. 282, 53-67.

1977 On the $(p, q)$-type and lower $(p, q)$-type of an entire function. J. Reine Angew. Math. 290, 180-190.

Kiselman, Christer O.

1977 Fonctions delta-convexes, delta-sousharmoniques et delta-plurisousharmoniques. Séminaire Pierre Lelong (Analyse) Année 1975/766; Lecture Notes in Mathematics 578, 93-107. Springer-Verlag.

1978 The partial Legendre transformation for plurisubharmonic functions. Inventiones Math. 49, 137-148.

1979 Densité des fonctions plurisousharmoniques. Bull. Soc. Math. France 107, 295304.

1981 The growth of restrictions of plurisubharmonic functions. Mathematical Analysis and Applications, Part B, L. Nachbin (Ed.). Advances in Mathematics Supplementary Studies, vol. 7B, 435-454.

1983 The use of conjugate convex functions in complex analysis. Complex Analysis, J. Ławrynowicz and J. Siciak (Eds.). Banach Center Publications, vol. 11, 131-142.

1984 Croissance des fonctions plurisousharmoniques en dimension infinie. Ann. Inst. Fourier Grenoble 34, 155-183.

1986 Konvekseco en kompleksa analitiko unu-dimensia. Lecture Notes 1986:LN2. Uppsala: Uppsala University, Department of Mathematics. 
1987 Un nombre de Lelong raffiné. Séminaire d'Analyse Complexe et Géométrie 198587, 61-70. Faculté des Sciences de Tunis \& Faculté des Sciences et Techniques de Monastir.

1992 La teoremo de Siu por abstraktaj nombroj de Lelong. Aktoj de Internacia Scienca Akademio Comenius, vol. 1, pp. 56-65. Beijing.

1993 Order and type as measures of growth for convex or entire functions. Proc. London Math. Soc. (3) 66, 152-186.

Kjellberg, Bo

1974 The convexity theorem of Hadamard-Hayman. Proceedings of the symposium in mathematics at the Royal Institute of Technology in June 1973, 87-114. Stockholm: The Royal Institute of Technology.

Klimek, Maciej

1991 Pluripotential Theory. Oxford: Oxford University Press.

Lelong, Pierre

1969 Plurisubharmonic functions and positive differential forms. London: Gordon and Breach.

1985 Les objets souples de l'analyse complexe. Exposition. Math. 3, 149-164.

Lelong, Pierre, and Gruman, Lawrence

1986 Entire Functions of Several Variables. Springer-Verlag.

Riihentaus, Juhani

1989 On a theorem of Avanissian-Arsove. Exposition. Math., 7, 69-72.

Rockafellar, R. T.

1970 Convex Analysis. Princeton: Princeton University Press.

Sato, D.

1963 On the rate of growth of entire functions of fast growth. Bull. Amer. Math. Soc. 69, 411-414.

Siu, Yum-Tong

1974 Analyticity of sets associated the Lelong numbers and the extension of closed positive currents. Inventiones Math. 27, 53-156.

Skoda, Henri

1972 Sous-ensemble analytiques d'ordre fini ou infini dans $\mathbf{C}^{n}$. Bull. Soc. Math. France 100, 353-408.

WANG Xiaoqin

1991 Analyticity theorems for parameter-dependent currents. Math. Scand. 69, 179198.

Wiegerinck, J.

1988 Separately subharmonic functions need not be subharmonic. Proc. Amer. Math. Soc. 104, 770-771. 


\section{Index}

addition, lower 31

addition, upper 31

coefficient function 36

complex Hessian matrix 11

conjugate function 33

convex 4,5

convolution 7

$\bar{\partial}$ equation 23

density 18

distribution 6

effective domain 33

epigraph 5

epigraph, strict 5

epigraphical sum 35

Fenchel transform 33

Fenchel transformation 33

fundamental solution 6

growth function 30,35

Harnack's inequality 20

Hessian form 11

Hessian matrix 10

Hörmander-Bombieri theorem 23

infimal convolution 35

integrability index 23

Laplacian 6

Legendre transform 33

Lelong number 18, 20

Levi form 11

Levi matrix 11

lower addition 31

lower semicontinuity 33

marginal function 9

mean-value inequality 4

Minimum Principle 16

Monge-Ampre operator 15

order, relative 30

plurisubharmonic 5

polar set 25

pseudoconvex 9

quasi-inverse 13

radial 7

relative order 30

relative type 32

semicontinuity, lower 33

semicontinuity, upper 5

strict epigraph 5 
strongly convex 17

Siu's theorem 28

subharmonic 4

superlevel set 23

test function 6

transpose 10

type, relative 32

upper addition 31

upper semicontinuity 5 